\title{
Palladium-Catalyzed Synthesis of Spiro[2.4]heptanes: Ligand-Dependent Position- Control in the Nucleophilic Attack to a $\pi$-Allylpalladium Intermediate
}

\author{
Ryo Shintani,* Soyoung Park, and Tamio Hayashi* \\ Department of Chemistry, Graduate School of Science, Kyoto University, \\ Sakyo, Kyoto 606-8502, Japan
}

\section{Supporting Information}

\section{General}

All air- and moisture-sensitive manipulations were carried out with standard Schlenk techniques under nitrogen or in a glove box under argon.

$\mathrm{CH}_{2} \mathrm{Cl}_{2}$ and 1,2-dichloroethane were distilled over $\mathrm{CaH}_{2}$ under nitrogen.

Methyl acrylate (Wako Chemicals), ethyl acrylate (Kanto Chemicals), tert-butyl acrylate (Wako Chemicals), acrylonitrile (Aldrich), 2-cyclopenten-1-one (Zeon), 2(5H)-furanone (Aldrich), triphenylphosphine (Wako Chemicals), dppf (Fluka), trimethyl phosphite (TCI), triisopropyl phosphite (TCI), 2-(di-tert-butylphosphino)biphenyl (Wako Chemicals), and tri$o$-tolylphosphine (Kanto Chemicals) were used as received. $\mathbf{1 a}-\mathbf{1 e},{ }^{1} \mathrm{PdCp}\left(\eta^{3}-\mathrm{C}_{3} \mathrm{H}_{5}\right),{ }^{2}$ and binap $^{3}$ were synthesized following the literature procedures.

All other chemicals and solvents were purchased from Aldrich, Wako Chemicals, TCI, or Kanto Chemicals and used as received.

\section{Catalytic Reactions}

\section{General Procedure for Table 2.}

A solution of $\mathrm{PdCp}\left(\eta^{3}-\mathrm{C}_{3} \mathrm{H}_{5}\right)(3.2 \mathrm{mg}, 15 \mu \mathrm{mol}), \mathrm{P}(\mathrm{O} i-\mathrm{Pr})_{3}(7.4 \mu \mathrm{L}, 30 \mu \mathrm{mol})$, compound $1(0.54 \mathrm{mmol})$, and electron-deficient olefin $2(0.30 \mathrm{mmol})$ in $\mathrm{CH}_{2} \mathrm{Cl}_{2}(1.5 \mathrm{~mL})$ was stirred for $24 \mathrm{~h}$ at $40{ }^{\circ} \mathrm{C}$. The reaction mixture was directly passed through a pad of silica gel with EtOAc and the solvent was removed under vacuum. The residue was purified by silica gel preparative TLC to afford cycloadducts.

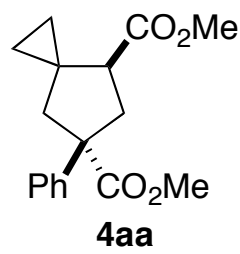

Entry 1. $86 \%$ yield $(\mathrm{dr}=79 / 21)$ along with $5 \%$ yield of 3aa. The relative configuration was assigned by analogy with 4ea (entry 5 ).

Major diastereomer of 4aa: ${ }^{1} \mathrm{H}$ NMR $\left(\mathrm{CDCl}_{3}\right): \delta$ 7.34-7.29 $(\mathrm{m}, 4 \mathrm{H}), 7.26-7.23(\mathrm{~m}, 1 \mathrm{H})$, $3.650(\mathrm{~s}, 3 \mathrm{H}), 3.649(\mathrm{~s}, 3 \mathrm{H}), 3.25\left(\mathrm{ddd},{ }^{2} J_{\mathrm{HH}}=13.1 \mathrm{~Hz}\right.$ and ${ }^{3} J_{\mathrm{HH}}=8.2 \mathrm{~Hz}$ and ${ }^{4} J_{\mathrm{HH}}=2.4 \mathrm{~Hz}$,

\footnotetext{
${ }^{1}$ Shintani, R.; Murakami, M.; Hayashi, T. J. Am. Chem. Soc. 2007, 129, asap.

${ }^{2}$ Parker, G.; Werner, H. Helv. Chim. Acta 1973, 56, 2819.

${ }^{3}$ Cai, D.; Payack, J. F.; Bender, D. R.; Hughes, D. L.; Verhoeven, T. R.; Reider, P. J. J. Org. Chem. 1994, 59, 7180.
} 
$1 \mathrm{H}), 2.74\left(\mathrm{t},{ }^{3} J_{\mathrm{HH}}=8.3 \mathrm{~Hz}, 1 \mathrm{H}\right), 2.51\left(\mathrm{~d},{ }^{2} J_{\mathrm{HH}}=12.1 \mathrm{~Hz}, 1 \mathrm{H}\right), 2.35\left(\mathrm{dd},{ }^{2} J_{\mathrm{HH}}=12.9 \mathrm{~Hz}\right.$ and $\left.{ }^{3} J_{\mathrm{HH}}=8.4 \mathrm{~Hz}, 1 \mathrm{H}\right), 2.25\left(\mathrm{dd},{ }^{2} J_{\mathrm{HH}}=12.2 \mathrm{~Hz}\right.$ and $\left.{ }^{4} J_{\mathrm{HH}}=2.3 \mathrm{~Hz}, 1 \mathrm{H}\right), 0.66-0.60(\mathrm{~m}, 2 \mathrm{H}), 0.59-$ $0.52(\mathrm{~m}, 2 \mathrm{H}) .{ }^{13} \mathrm{C} \mathrm{NMR}\left(\mathrm{CDCl}_{3}\right): \delta 176.0,175.6,142.2,128.5,127.2,126.7,58.7,52.5,51.7$, 49.0, 45.0, 40.0, 24.7, 17.2, 7.7. Anal. Calcd for $\mathrm{C}_{17} \mathrm{H}_{20} \mathrm{O}_{4}: \mathrm{C}, 70.81 ; \mathrm{H}, 6.99$. Found: C, $71.10 ; \mathrm{H}, 6.99$.

Minor diastereomer of 4aa: ${ }^{1} \mathrm{H}$ NMR $\left(\mathrm{CDCl}_{3}\right): \delta 7.39\left(\mathrm{~d},{ }^{3} J_{\mathrm{HH}}=7.2 \mathrm{~Hz}, 2 \mathrm{H}\right), 7.33\left(\mathrm{t},{ }^{3} J_{\mathrm{HH}}\right.$ $=7.3 \mathrm{~Hz}, 2 \mathrm{H}), 7.25\left(\mathrm{tt},{ }^{3} J_{\mathrm{HH}}=7.3 \mathrm{~Hz}\right.$ and $\left.{ }^{4} J_{\mathrm{HH}}=1.2 \mathrm{~Hz}, 1 \mathrm{H}\right), 3.64(\mathrm{~s}, 6 \mathrm{H}), 3.07\left(\mathrm{dd},{ }^{2} J_{\mathrm{HH}}=\right.$ $13.5 \mathrm{~Hz}$ and $\left.{ }^{3} J_{\mathrm{HH}}=7.7 \mathrm{~Hz}, 1 \mathrm{H}\right), 2.78\left(\mathrm{dd},{ }^{3} J_{\mathrm{HH}}=8.1\right.$ and $\left.7.8 \mathrm{~Hz}, 1 \mathrm{H}\right), 2.70\left(\mathrm{~d},{ }^{2} J_{\mathrm{HH}}=13.1 \mathrm{~Hz}\right.$, $1 \mathrm{H}), 2.59\left(\mathrm{ddd},{ }^{2} J_{\mathrm{HH}}=13.5 \mathrm{~Hz}\right.$ and ${ }^{3} J_{\mathrm{HH}}=8.4 \mathrm{~Hz}$ and $\left.{ }^{4} J_{\mathrm{HH}}=1.2 \mathrm{~Hz}, 1 \mathrm{H}\right), 2.12\left(\mathrm{dd},{ }^{2} J_{\mathrm{HH}}=\right.$ $13.1 \mathrm{~Hz}$ and $\left.{ }^{4} J_{\mathrm{HH}}=1.2 \mathrm{~Hz}, 1 \mathrm{H}\right), 0.56-0.49(\mathrm{~m}, 4 \mathrm{H}) .{ }^{13} \mathrm{C} \mathrm{NMR}\left(\mathrm{CDCl}_{3}\right): \delta 175.6,174.4,142.7$, 128.6, 127.1, 126.6, 57.8, 52.5, 51.7, 48.6, 45.7, 39.5, 24.0, 14.2, 10.2. Anal. Calcd for $\mathrm{C}_{17} \mathrm{H}_{20} \mathrm{O}_{4}$ : C, 70.81; H, 6.99. Found: C, 70.82; H, 7.15.

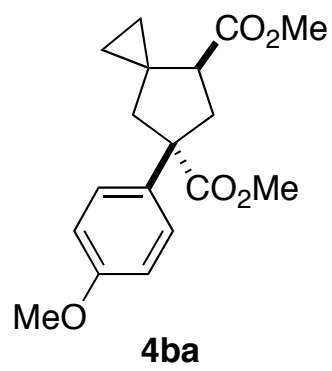

Entry 2. $91 \%$ yield $(\mathrm{dr}=74 / 26)$ along with $7 \%$ yield of $\mathbf{3 b a}$. The relative configuration was assigned by analogy with 4ea (entry 5 ).

Major diastereomer of 4ba: ${ }^{1} \mathrm{H}$ NMR $\left(\mathrm{CDCl}_{3}\right): \delta 7.25\left(\mathrm{~d},{ }^{3} J_{\mathrm{HH}}=8.9 \mathrm{~Hz}, 2 \mathrm{H}\right), 6.84\left(\mathrm{~d},{ }^{3} J_{\mathrm{HH}}\right.$ $=8.9 \mathrm{~Hz}, 2 \mathrm{H}), 3.79(\mathrm{~s}, 3 \mathrm{H}), 3.65(\mathrm{~s}, 3 \mathrm{H}), 3.64(\mathrm{~s}, 3 \mathrm{H}), 3.22\left(\mathrm{ddd},{ }^{2} J_{\mathrm{HH}}=12.8 \mathrm{~Hz}\right.$ and ${ }^{3} J_{\mathrm{HH}}=$ $8.2 \mathrm{~Hz}$ and $\left.{ }^{4} J_{\mathrm{HH}}=2.3 \mathrm{~Hz}, 1 \mathrm{H}\right), 2.73\left(\mathrm{t},{ }^{3} J_{\mathrm{HH}}=8.4 \mathrm{~Hz}, 1 \mathrm{H}\right), 2.46\left(\mathrm{~d},{ }^{2} J_{\mathrm{HH}}=12.1 \mathrm{~Hz}, 1 \mathrm{H}\right), 2.32$ $\left(\mathrm{dd},{ }^{2} J_{\mathrm{HH}}=12.9 \mathrm{~Hz}\right.$ and $\left.{ }^{3} J_{\mathrm{HH}}=8.5 \mathrm{~Hz}, 1 \mathrm{H}\right), 2.23\left(\mathrm{dd},{ }^{2} J_{\mathrm{HH}}=12.1 \mathrm{~Hz}\right.$ and $\left.{ }^{4} J_{\mathrm{HH}}=2.2 \mathrm{~Hz}, 1 \mathrm{H}\right)$, 0.65-0.58 (m, 2H), 0.58-0.50 (m, 2H). ${ }^{13} \mathrm{C} \mathrm{NMR}\left(\mathrm{CDCl}_{3}\right): \delta 176.1,175.5,158.7,134.5$, $127.8,113.8,57.9,55.3,52.4,51.6,49.0,45.2,40.1,24.6,17.1,7.7$. Anal. Calcd for $\mathrm{C}_{18} \mathrm{H}_{22} \mathrm{O}_{5}$ : C, 67.91; H, 6.97. Found: C, 68.11; H, 7.01.

Minor diastereomer of $4 \mathrm{ba}:{ }^{1} \mathrm{H}$ NMR $\left(\mathrm{CDCl}_{3}\right): \delta 7.31\left(\mathrm{~d},{ }^{3} J_{\mathrm{HH}}=8.9 \mathrm{~Hz}, 2 \mathrm{H}\right), 6.86\left(\mathrm{~d},{ }^{3} J_{\mathrm{HH}}\right.$ $=8.9 \mathrm{~Hz}, 2 \mathrm{H}), 3.80(\mathrm{~s}, 3 \mathrm{H}), 3.641(\mathrm{~s}, 3 \mathrm{H}), 3.636(\mathrm{~s}, 3 \mathrm{H}), 3.03\left(\mathrm{dd},{ }^{2} J_{\mathrm{HH}}=13.4 \mathrm{~Hz}\right.$ and ${ }^{3} J_{\mathrm{HH}}=$ $7.8 \mathrm{~Hz}, 1 \mathrm{H}), 2.77\left(\mathrm{t},{ }^{3} J_{\mathrm{HH}}=8.0 \mathrm{~Hz}, 1 \mathrm{H}\right), 2.67\left(\mathrm{~d},{ }^{2} J_{\mathrm{HH}}=13.1 \mathrm{~Hz}, 1 \mathrm{H}\right), 2.56\left(\mathrm{ddd},{ }^{2} J_{\mathrm{HH}}=13.4\right.$ $\mathrm{Hz}$ and ${ }^{3} J_{\mathrm{HH}}=8.2 \mathrm{~Hz}$ and $\left.{ }^{4} J_{\mathrm{HH}}=1.2 \mathrm{~Hz}, 1 \mathrm{H}\right), 2.09\left(\mathrm{dd},{ }^{2} J_{\mathrm{HH}}=13.1 \mathrm{~Hz}\right.$ and $\left.{ }^{4} J_{\mathrm{HH}}=1.2 \mathrm{~Hz}, 1 \mathrm{H}\right)$, 0.54-0.48 (m, 4H). ${ }^{13} \mathrm{C} \mathrm{NMR}\left(\mathrm{CDCl}_{3}\right): \delta 175.8,174.5,158.6,134.6,127.7,113.9,57.1,55.4$, 52.4, 51.6, 48.5, 45.7, 39.6, 23.9, 14.3, 10.2. Anal. Calcd for $\mathrm{C}_{18} \mathrm{H}_{22} \mathrm{O}_{5}: \mathrm{C}, 67.91 ; \mathrm{H}, 6.97$. Found: C, 67.93; H, 6.84.

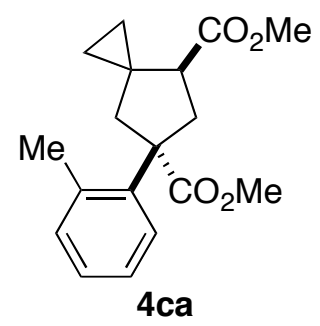

Entry 3. $91 \%$ yield $(\mathrm{dr}=77 / 23)$ along with $6 \%$ yield of 3 ca. The relative configuration was assigned by analogy with 4ea (entry 5 ).

Major diastereomer of $\mathbf{4} \mathbf{c a}:{ }^{1} \mathrm{H}$ NMR $\left(\mathrm{CDCl}_{3}\right): \delta$ 7.38-7.35 $(\mathrm{m}, 1 \mathrm{H}), 7.20-7.15(\mathrm{~m}, 2 \mathrm{H})$, 
7.12-7.10 (m, $1 \mathrm{H}), 3.641(\mathrm{~s}, 3 \mathrm{H}), 3.636(\mathrm{~s}, 3 \mathrm{H}), 3.30\left(\mathrm{ddd},{ }^{2} J_{\mathrm{HH}}=13.1 \mathrm{~Hz}\right.$ and ${ }^{3} J_{\mathrm{HH}}=8.3 \mathrm{~Hz}$ and $\left.{ }^{4} J_{\mathrm{HH}}=2.2 \mathrm{~Hz}, 1 \mathrm{H}\right), 2.84\left(\mathrm{t},{ }^{3} J_{\mathrm{HH}}=8.2 \mathrm{~Hz}, 1 \mathrm{H}\right), 2.72\left(\mathrm{~d},{ }^{2} J_{\mathrm{HH}}=12.2 \mathrm{~Hz}, 1 \mathrm{H}\right), 2.32(\mathrm{~s}, 3 \mathrm{H})$, $2.30\left(\mathrm{dd},{ }^{2} J_{\mathrm{HH}}=13.0 \mathrm{~Hz}\right.$ and $\left.{ }^{3} J_{\mathrm{HH}}=8.0 \mathrm{~Hz}, 1 \mathrm{H}\right), 2.18\left(\mathrm{dd},{ }^{2} J_{\mathrm{HH}}=12.2 \mathrm{~Hz}\right.$ and ${ }^{4} J_{\mathrm{HH}}=2.2 \mathrm{~Hz}$, $1 \mathrm{H}), 0.64-0.53(\mathrm{~m}, 4 \mathrm{H}) .{ }^{13} \mathrm{C} \mathrm{NMR}\left(\mathrm{CDCl}_{3}\right): \delta 176.4,175.6,140.6,136.7,131.8,127.3,127.0$, 126.1, 58.4, 52.4, 51.6, 49.0, 46.1, 39.2, 23.9, 21.2, 16.7, 7.5. Anal. Calcd for $\mathrm{C}_{18} \mathrm{H}_{22} \mathrm{O}_{4}$ : C, 71.50; H, 7.33. Found: C, 71.61; H, 7.28.

Minor diastereomer of 4ca: ${ }^{1} \mathrm{H}$ NMR $\left(\mathrm{CDCl}_{3}\right): \delta 7.39\left(\mathrm{dd},{ }^{3} J_{\mathrm{HH}}=7.0 \mathrm{~Hz}\right.$ and ${ }^{4} J_{\mathrm{HH}}=2.1$ $\mathrm{Hz}, 1 \mathrm{H}), 7.22-7.14(\mathrm{~m}, 3 \mathrm{H}), 3.66(\mathrm{~s}, 3 \mathrm{H}), 3.65(\mathrm{~s}, 3 \mathrm{H}), 3.05\left(\mathrm{dd},{ }^{2} J_{\mathrm{HH}}=13.4 \mathrm{~Hz}\right.$ and ${ }^{3} J_{\mathrm{HH}}=9.7$ $\mathrm{Hz}, 1 \mathrm{H}), 2.86\left(\mathrm{dd},{ }^{3} J_{\mathrm{HH}}=9.6\right.$ and $\left.7.6 \mathrm{~Hz}, 1 \mathrm{H}\right), 2.70\left(\mathrm{~d},{ }^{2} J_{\mathrm{HH}}=13.2 \mathrm{~Hz}, 1 \mathrm{H}\right), 2.53\left(\mathrm{ddd},{ }^{2} J_{\mathrm{HH}}=\right.$ $13.4 \mathrm{~Hz}$ and ${ }^{3} J_{\mathrm{HH}}=7.7 \mathrm{~Hz}$ and $\left.{ }^{4} J_{\mathrm{HH}}=1.7 \mathrm{~Hz}, 1 \mathrm{H}\right), 2.21(\mathrm{~s}, 3 \mathrm{H}), 2.08\left(\mathrm{dd},{ }^{2} J_{\mathrm{HH}}=13.2 \mathrm{~Hz}\right.$ and $\left.{ }^{4} J_{\mathrm{HH}}=1.6 \mathrm{~Hz}, 1 \mathrm{H}\right), 0.59-0.57(\mathrm{~m}, 2 \mathrm{H}), 0.50-0.48(\mathrm{~m}, 2 \mathrm{H}) .{ }^{13} \mathrm{C} \mathrm{NMR}\left(\mathrm{CDCl}_{3}\right): \delta 176.6,174.0$, 140.9, 136.2, 132.0, 127.1, 126.5, 125.9, 57.1, 52.6, 51.7, 48.7, 46.5, 39.9, 23.9, 20.5, 14.0, 10.6. Anal. Calcd for $\mathrm{C}_{18} \mathrm{H}_{22} \mathrm{O}_{4}$ : C, 71.50; H, 7.33. Found: C, 71.38; H, 7.34.

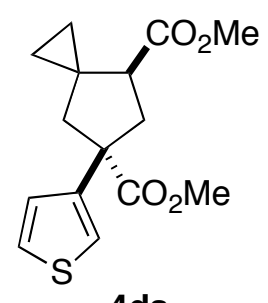

4da

Entry 4. $87 \%$ yield $(\mathrm{dr}=72 / 28)$ along with $7 \%$ yield of $\mathbf{3 d a}$. The relative configuration was assigned by analogy with 4ea (entry 5 ).

Major diastereomer of 4da: ${ }^{1} \mathrm{H}$ NMR $\left(\mathrm{CDCl}_{3}\right): \delta 7.25\left(\mathrm{dd},{ }^{3} J_{\mathrm{HH}}=5.0 \mathrm{~Hz}\right.$ and ${ }^{4} J_{\mathrm{HH}}=2.9$ $\mathrm{Hz}, 1 \mathrm{H}), 7.11\left(\mathrm{dd},{ }^{4} J_{\mathrm{HH}}=2.9\right.$ and $\left.1.3 \mathrm{~Hz}, 1 \mathrm{H}\right), 7.05\left(\mathrm{dd},{ }^{3} J_{\mathrm{HH}}=5.0 \mathrm{~Hz}\right.$ and $\left.{ }^{4} J_{\mathrm{HH}}=1.3 \mathrm{~Hz}, 1 \mathrm{H}\right)$, $3.68(\mathrm{~s}, 3 \mathrm{H}), 3.66(\mathrm{~s}, 3 \mathrm{H}), 3.16\left(\mathrm{ddd},{ }^{2} J_{\mathrm{HH}}=12.9 \mathrm{~Hz}\right.$ and ${ }^{3} J_{\mathrm{HH}}=8.2 \mathrm{~Hz}$ and $\left.{ }^{4} J_{\mathrm{HH}}=2.2 \mathrm{~Hz}, 1 \mathrm{H}\right)$, $2.72\left(\mathrm{t},{ }^{3} J_{\mathrm{HH}}=8.3 \mathrm{~Hz}, 1 \mathrm{H}\right), 2.46\left(\mathrm{~d},{ }^{2} J_{\mathrm{HH}}=12.2 \mathrm{~Hz}, 1 \mathrm{H}\right), 2.39\left(\mathrm{dd},{ }^{2} J_{\mathrm{HH}}=13.1 \mathrm{~Hz}\right.$ and ${ }^{3} J_{\mathrm{HH}}=$ $8.4 \mathrm{~Hz}, 1 \mathrm{H}), 2.22\left(\mathrm{dd},{ }^{2} J_{\mathrm{HH}}=12.2 \mathrm{~Hz}\right.$ and $\left.{ }^{4} J_{\mathrm{HH}}=2.2 \mathrm{~Hz}, 1 \mathrm{H}\right), 0.64-0.58(\mathrm{~m}, 2 \mathrm{H}), 0.57-0.51$ $(\mathrm{m}, 2 \mathrm{H}) .{ }^{13} \mathrm{C} \mathrm{NMR}\left(\mathrm{CDCl}_{3}\right): \delta 175.5,175.4,142.9,126.7,125.7,120.8,56.0,52.5,51.7$, 49.0, 45.9, 40.0, 24.7, 16.9, 7.7. Anal. Calcd for $\mathrm{C}_{15} \mathrm{H}_{18} \mathrm{O}_{4} \mathrm{~S}: \mathrm{C}, 61.20 ; \mathrm{H}, 6.16$. Found: $\mathrm{C}$, $61.38 ; \mathrm{H}, 6.01$.

Minor diastereomer of 4da: ${ }^{1} \mathrm{H}$ NMR $\left(\mathrm{CDCl}_{3}\right): \delta 7.27\left(\mathrm{dd},{ }^{3} J_{\mathrm{HH}}=5.0 \mathrm{~Hz}\right.$ and ${ }^{4} J_{\mathrm{HH}}=2.9$ $\mathrm{Hz}, 1 \mathrm{H}), 7.16\left(\mathrm{dd},{ }^{4} J_{\mathrm{HH}}=2.9\right.$ and $\left.1.3 \mathrm{~Hz}, 1 \mathrm{H}\right), 7.08\left(\mathrm{dd},{ }^{3} J_{\mathrm{HH}}=5.0 \mathrm{~Hz}\right.$ and $\left.{ }^{4} J_{\mathrm{HH}}=1.3 \mathrm{~Hz}, 1 \mathrm{H}\right)$, $3.67(\mathrm{~s}, 3 \mathrm{H}), 3.64(\mathrm{~s}, 3 \mathrm{H}), 2.97\left(\mathrm{dd},{ }^{2} J_{\mathrm{HH}}=13.4 \mathrm{~Hz}\right.$ and $\left.{ }^{3} J_{\mathrm{HH}}=8.2 \mathrm{~Hz}, 1 \mathrm{H}\right), 2.75\left(\mathrm{t},{ }^{3} J_{\mathrm{HH}}=8.1\right.$ $\mathrm{Hz}, 1 \mathrm{H}), 2.69\left(\mathrm{~d},{ }^{2} J_{\mathrm{HH}}=13.1 \mathrm{~Hz}, 1 \mathrm{H}\right), 2.57\left(\mathrm{ddd},{ }^{2} J_{\mathrm{HH}}=13.4 \mathrm{~Hz}\right.$ and ${ }^{3} J_{\mathrm{HH}}=8.1 \mathrm{~Hz}$ and ${ }^{4} J_{\mathrm{HH}}=$ $1.5 \mathrm{~Hz}, 1 \mathrm{H}), 2.05\left(\mathrm{dd},{ }^{2} J_{\mathrm{HH}}=13.1 \mathrm{~Hz}\right.$ and $\left.{ }^{4} J_{\mathrm{HH}}=1.5 \mathrm{~Hz}, 1 \mathrm{H}\right), 0.56-0.49(\mathrm{~m}, 4 \mathrm{H}) .{ }^{13} \mathrm{C} \mathrm{NMR}$ $\left(\mathrm{CDCl}_{3}\right): \delta 175.1,174.5,143.6,127.0,125.9,121.1,55.2,52.5,51.7,48.7,46.0,40.1,24.0$, 15.0, 9.7. Anal. Calcd for $\mathrm{C}_{15} \mathrm{H}_{18} \mathrm{O}_{4} \mathrm{~S}$ : C, 61.20; H, 6.16. Found: C, 61.14; H, 6.22.

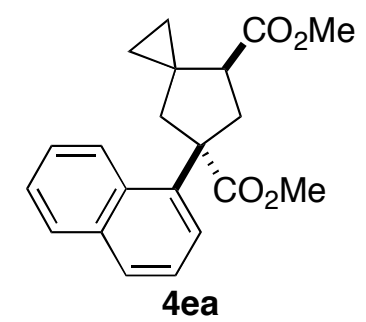

Entry 5. 97\% yield $(\mathrm{dr}=70 / 30)$ along with $3 \%$ yield of 3ea. The relative configuration was determined by X-ray crystallographic analysis of the major diastereomer. 
Major diastereomer of 4ea: ${ }^{1} \mathrm{H}$ NMR $\left(\mathrm{CDCl}_{3}\right): \delta 8.12-8.09(\mathrm{~m}, 1 \mathrm{H}), 7.86-7.84(\mathrm{~m}, 1 \mathrm{H})$, $7.78\left(\mathrm{~d},{ }^{3} J_{\mathrm{HH}}=8.2 \mathrm{~Hz}, 1 \mathrm{H}\right), 7.58\left(\mathrm{dd},{ }^{3} J_{\mathrm{HH}}=7.3 \mathrm{~Hz}\right.$ and $\left.{ }^{4} J_{\mathrm{HH}}=1.0 \mathrm{~Hz}, 1 \mathrm{H}\right), 7.48-7.43(\mathrm{~m}, 3 \mathrm{H})$, $3.63(\mathrm{~s}, 3 \mathrm{H}), 3.58(\mathrm{~s}, 3 \mathrm{H}), 3.49\left(\mathrm{ddd},{ }^{2} J_{\mathrm{HH}}=13.3 \mathrm{~Hz}\right.$ and ${ }^{3} J_{\mathrm{HH}}=8.2 \mathrm{~Hz}$ and $\left.{ }^{4} J_{\mathrm{HH}}=2.1 \mathrm{~Hz}, 1 \mathrm{H}\right)$, $2.98\left(\mathrm{t},{ }^{3} J_{\mathrm{HH}}=8.1 \mathrm{~Hz}, 1 \mathrm{H}\right), 2.88\left(\mathrm{~d},{ }^{2} J_{\mathrm{HH}}=12.2 \mathrm{~Hz}, 1 \mathrm{H}\right), 2.43\left(\mathrm{dd},{ }^{2} J_{\mathrm{HH}}=13.3 \mathrm{~Hz}\right.$ and ${ }^{3} J_{\mathrm{HH}}=$ $8.1 \mathrm{~Hz}, 1 \mathrm{H}), 2.34\left(\mathrm{dd},{ }^{2} J_{\mathrm{HH}}=12.2 \mathrm{~Hz}\right.$ and $\left.{ }^{4} J_{\mathrm{HH}}=2.0 \mathrm{~Hz}, 1 \mathrm{H}\right), 0.70-0.63(\mathrm{~m}, 3 \mathrm{H}), 0.59-0.55$ $(\mathrm{m}, 1 \mathrm{H}) .{ }^{13} \mathrm{C} \mathrm{NMR}\left(\mathrm{CDCl}_{3}\right): \delta 177.2,175.4,138.7,134.4,132.1,129.2,128.4,126.1,125.44$, 125.38, 124.8, 124.0, 58.2, 52.6, 51.6, 49.2, 46.4, 40.3, 23.9, 16.4, 7.8. Anal. Calcd for $\mathrm{C}_{21} \mathrm{H}_{22} \mathrm{O}_{4}$ : C, 74.54; H, 6.55. Found: C, 74.67; H, 6.61.

Minor diastereomer of 4ea: ${ }^{1} \mathrm{H}$ NMR $\left(\mathrm{CDCl}_{3}\right): \delta 7.92-7.89(\mathrm{~m}, 1 \mathrm{H}), 7.88-7.85(\mathrm{~m}, 1 \mathrm{H})$, $7.79\left(\mathrm{~d},{ }^{3} J_{\mathrm{HH}}=8.3 \mathrm{~Hz}, 1 \mathrm{H}\right), 7.59\left(\mathrm{dd},{ }^{3} J_{\mathrm{HH}}=7.2 \mathrm{~Hz}\right.$ and $\left.{ }^{4} J_{\mathrm{HH}}=1.0 \mathrm{~Hz}, 1 \mathrm{H}\right), 7.48-7.44(\mathrm{~m}, 3 \mathrm{H})$, $3.66(\mathrm{~s}, 3 \mathrm{H}), 3.57(\mathrm{~s}, 3 \mathrm{H}), 3.18\left(\mathrm{dd},{ }^{2} J_{\mathrm{HH}}=13.1 \mathrm{~Hz}\right.$ and $\left.{ }^{3} J_{\mathrm{HH}}=9.5 \mathrm{~Hz}, 1 \mathrm{H}\right), 2.92\left(\mathrm{~d},{ }^{2} J_{\mathrm{HH}}=\right.$ $13.3 \mathrm{~Hz}, 1 \mathrm{H}), 2.88\left(\mathrm{dd},{ }^{3} J_{\mathrm{HH}}=9.5\right.$ and $\left.7.6 \mathrm{~Hz}, 1 \mathrm{H}\right), 2.81\left(\mathrm{ddd},{ }^{2} J_{\mathrm{HH}}=13.1 \mathrm{~Hz}\right.$ and ${ }^{3} J_{\mathrm{HH}}=7.6$ $\mathrm{Hz}$ and $\left.{ }^{4} J_{\mathrm{HH}}=1.5 \mathrm{~Hz}, 1 \mathrm{H}\right), 2.30\left(\mathrm{dd},{ }^{2} J_{\mathrm{HH}}=13.1 \mathrm{~Hz}\right.$ and $\left.{ }^{4} J_{\mathrm{HH}}=1.3 \mathrm{~Hz}, 1 \mathrm{H}\right), 0.61-0.58(\mathrm{~m}$, $1 \mathrm{H}), 0.54-0.48(\mathrm{~m}, 2 \mathrm{H}), 0.43-0.41(\mathrm{~m}, 1 \mathrm{H}) .{ }^{13} \mathrm{C} \mathrm{NMR}\left(\mathrm{CDCl}_{3}\right): \delta 177.2,174.1,138.2,134.7$, 131.3, 129.4, 128.4, 126.2, 125.5, 125.1, 124.5, 124.2, 57.1, 52.7, 51.7, 48.7, 46.8, 40.3, 23.8, 14.5, 10.1. Anal. Calcd for $\mathrm{C}_{21} \mathrm{H}_{22} \mathrm{O}_{4}$ : C, 74.54; H, 6.55. Found: C, 74.26; H, 6.65.

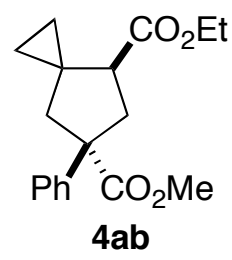

Entry 6. $92 \%$ yield $(\mathrm{dr}=79 / 21)$ along with $7 \%$ yield of $\mathbf{3 a b}$. The relative configuration was assigned by analogy with 4ea (entry 5).

Major diastereomer of 4ab: ${ }^{1} \mathrm{H}$ NMR $\left(\mathrm{CDCl}_{3}\right): \delta 7.35-7.29(\mathrm{~m}, 4 \mathrm{H}), 7.24\left(\mathrm{tt},{ }^{3} J_{\mathrm{HH}}=7.4\right.$ $\mathrm{Hz}$ and $\left.{ }^{4} J_{\mathrm{HH}}=1.6 \mathrm{~Hz}, 1 \mathrm{H}\right), 4.16\left(\mathrm{q},{ }^{3} J_{\mathrm{HH}}=7.1 \mathrm{~Hz}, 2 \mathrm{H}\right), 3.65(\mathrm{~s}, 3 \mathrm{H}), 3.24\left(\mathrm{ddd},{ }^{2} J_{\mathrm{HH}}=12.9\right.$ $\mathrm{Hz}$ and ${ }^{3} J_{\mathrm{HH}}=8.1 \mathrm{~Hz}$ and $\left.{ }^{4} J_{\mathrm{HH}}=2.3 \mathrm{~Hz}, 1 \mathrm{H}\right), 2.72\left(\mathrm{t},{ }^{3} J_{\mathrm{HH}}=8.2 \mathrm{~Hz}, 1 \mathrm{H}\right), 2.51\left(\mathrm{~d},{ }^{2} J_{\mathrm{HH}}=12.2\right.$ $\mathrm{Hz}, 1 \mathrm{H}), 2.35\left(\mathrm{dd},{ }^{2} J_{\mathrm{HH}}=12.9 \mathrm{~Hz}\right.$ and $\left.{ }^{3} J_{\mathrm{HH}}=8.5 \mathrm{~Hz}, 1 \mathrm{H}\right), 2.26\left(\mathrm{dd},{ }^{2} J_{\mathrm{HH}}=12.2 \mathrm{~Hz}\right.$ and ${ }^{4} J_{\mathrm{HH}}=$ $2.3 \mathrm{~Hz}, 1 \mathrm{H}), 1.23\left(\mathrm{t},{ }^{3} J_{\mathrm{HH}}=7.1 \mathrm{~Hz}, 3 \mathrm{H}\right), 0.68-0.62(\mathrm{~m}, 2 \mathrm{H}), 0.59-0.51(\mathrm{~m}, 2 \mathrm{H}) .{ }^{13} \mathrm{C} \mathrm{NMR}$ $\left(\mathrm{CDCl}_{3}\right): \delta 176.0,175.1,142.3,128.5,127.2,126.7,60.5,58.6,52.5,49.0,45.1,40.0,24.6$, 17.1, 14.5, 7.6. Anal. Calcd for $\mathrm{C}_{18} \mathrm{H}_{22} \mathrm{O}_{4}$ : C, 71.50; H, 7.33. Found: C, 71.67; H, 7.40.

Minor diastereomer of $\mathbf{4 a b}:{ }^{1} \mathrm{H}$ NMR $\left(\mathrm{CDCl}_{3}\right): \delta 7.39\left(\mathrm{~d},{ }^{3} J_{\mathrm{HH}}=7.2 \mathrm{~Hz}, 2 \mathrm{H}\right), 7.33\left(\mathrm{t},{ }^{3} J_{\mathrm{HH}}\right.$ $=7.3 \mathrm{~Hz}, 2 \mathrm{H}), 7.25\left(\mathrm{tt},{ }^{3} J_{\mathrm{HH}}=7.3 \mathrm{~Hz}\right.$ and $\left.{ }^{4} J_{\mathrm{HH}}=1.2 \mathrm{~Hz}, 1 \mathrm{H}\right), 4.11\left(\mathrm{q},{ }^{3} J_{\mathrm{HH}}=7.2 \mathrm{~Hz}, 2 \mathrm{H}\right), 3.64$ $(\mathrm{s}, 3 \mathrm{H}), 3.07\left(\mathrm{ddd},{ }^{2} J_{\mathrm{HH}}=13.5 \mathrm{~Hz}\right.$ and ${ }^{3} J_{\mathrm{HH}}=7.7 \mathrm{~Hz}$ and $\left.J_{\mathrm{HH}}=0.5 \mathrm{~Hz}, 1 \mathrm{H}\right), 2.76\left(\mathrm{dd},{ }^{3} J_{\mathrm{HH}}=\right.$ 8.1 and $7.9 \mathrm{~Hz}, 1 \mathrm{H}), 2.70\left(\mathrm{~d},{ }^{2} J_{\mathrm{HH}}=13.1 \mathrm{~Hz}, 1 \mathrm{H}\right), 2.59\left(\mathrm{ddd},{ }^{2} J_{\mathrm{HH}}=13.5 \mathrm{~Hz}\right.$ and ${ }^{3} J_{\mathrm{HH}}=8.3$ $\mathrm{Hz}$ and $\left.{ }^{4} J_{\mathrm{HH}}=1.3 \mathrm{~Hz}, 1 \mathrm{H}\right), 2.12\left(\mathrm{dd},{ }^{2} J_{\mathrm{HH}}=13.1 \mathrm{~Hz}\right.$ and $\left.{ }^{4} J_{\mathrm{HH}}=1.2 \mathrm{~Hz}, 1 \mathrm{H}\right), 1.24\left(\mathrm{t},{ }^{3} J_{\mathrm{HH}}=\right.$ $7.1 \mathrm{~Hz}, 3 \mathrm{H}), 0.59-0.55(\mathrm{~m}, 1 \mathrm{H}), 0.54-0.50(\mathrm{~m}, 3 \mathrm{H}) .{ }^{13} \mathrm{C} \mathrm{NMR}\left(\mathrm{CDCl}_{3}\right): \delta 175.7,173.9,142.8$, 128.6, 127.1, 126.6, 60.5, 57.8, 52.5, 48.5, 45.8, 39.5, 24.0, 14.5, 14.1, 10.1. Anal. Calcd for $\mathrm{C}_{18} \mathrm{H}_{22} \mathrm{O}_{4}$ : C, 71.50; H, 7.33. Found: C, 71.53; H, 7.40.

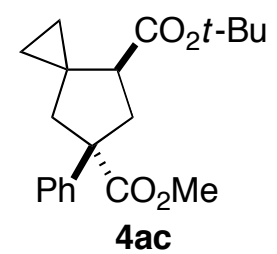

Entry 7. $77 \%$ yield $(\mathrm{dr}=90 / 10)$ along with $8 \%$ yield of 3ac. The relative configuration was assigned by analogy with 4ea (entry 5). 
Major diastereomer of 4ac: ${ }^{1} \mathrm{H}$ NMR $\left(\mathrm{CDCl}_{3}\right): \delta 7.33\left(\mathrm{~d},{ }^{3} J_{\mathrm{HH}}=7.1 \mathrm{~Hz}, 2 \mathrm{H}\right), 7.30\left(\mathrm{t},{ }^{3} J_{\mathrm{HH}}\right.$ $=7.1 \mathrm{~Hz}, 2 \mathrm{H}), 7.23\left(\mathrm{tt},{ }^{3} J_{\mathrm{HH}}=7.0 \mathrm{~Hz}\right.$ and $\left.{ }^{4} J_{\mathrm{HH}}=1.6 \mathrm{~Hz}, 1 \mathrm{H}\right), 3.64(\mathrm{~s}, 3 \mathrm{H}), 3.21\left(\mathrm{ddd},{ }^{2} J_{\mathrm{HH}}=\right.$ $12.9 \mathrm{~Hz}$ and ${ }^{3} J_{\mathrm{HH}}=8.1 \mathrm{~Hz}$ and $\left.{ }^{4} J_{\mathrm{HH}}=2.3 \mathrm{~Hz}, 1 \mathrm{H}\right), 2.60\left(\mathrm{t},{ }^{3} J_{\mathrm{HH}}=8.3 \mathrm{~Hz}, 1 \mathrm{H}\right), 2.49\left(\mathrm{~d},{ }^{2} J_{\mathrm{HH}}=\right.$ $12.1 \mathrm{~Hz}, 1 \mathrm{H}), 2.30\left(\mathrm{dd},{ }^{2} J_{\mathrm{HH}}=12.9 \mathrm{~Hz}\right.$ and $\left.{ }^{3} J_{\mathrm{HH}}=8.5 \mathrm{~Hz}, 1 \mathrm{H}\right), 2.24\left(\mathrm{dd},{ }^{2} J_{\mathrm{HH}}=12.2 \mathrm{~Hz}\right.$ and $\left.{ }^{4} J_{\mathrm{HH}}=2.2 \mathrm{~Hz}, 1 \mathrm{H}\right), 1.42(\mathrm{~s}, 9 \mathrm{H}), 0.75-0.71(\mathrm{~m}, 1 \mathrm{H}), 0.63-0.60(\mathrm{~m}, 1 \mathrm{H}), 0.58-0.50(\mathrm{~m}, 2 \mathrm{H})$. ${ }^{13} \mathrm{C}$ NMR $\left(\mathrm{CDCl}_{3}\right): \delta 176.1,174.4,142.5,128.5,127.1,126.7,80.5,58.6,52.4,49.8,45.1$, 40.1, 28.3, 24.6, 16.8, 7.5. Anal. Calcd for $\mathrm{C}_{20} \mathrm{H}_{26} \mathrm{O}_{4}$ : C, 72.70; H, 7.93. Found: C, 72.53; H, 7.88 .

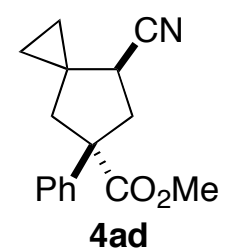

Entry 8. $88 \%$ yield $(\mathrm{dr}=76 / 24)$ along with $7 \%$ yield of 3ad. The relative configuration was assigned by analogy with 4ea (entry 5).

Major diastereomer of 4ad: ${ }^{1} \mathrm{H}$ NMR $\left(\mathrm{CDCl}_{3}\right)$ : $\delta$ 7.36-7.26 (m, 5H), $3.66(\mathrm{~s}, 3 \mathrm{H}), 3.44$ $\left(\mathrm{ddd},{ }^{2} J_{\mathrm{HH}}=12.9 \mathrm{~Hz}\right.$ and ${ }^{3} J_{\mathrm{HH}}=8.3 \mathrm{~Hz}$ and $\left.{ }^{4} J_{\mathrm{HH}}=2.2 \mathrm{~Hz}, 1 \mathrm{H}\right), 2.93\left(\mathrm{t},{ }^{3} J_{\mathrm{HH}}=8.7 \mathrm{~Hz}, 1 \mathrm{H}\right)$, $2.49\left(\mathrm{~d},{ }^{2} J_{\mathrm{HH}}=12.7 \mathrm{~Hz}, 1 \mathrm{H}\right), 2.41\left(\mathrm{dd},{ }^{2} J_{\mathrm{HH}}=12.7 \mathrm{~Hz}\right.$ and $\left.{ }^{4} J_{\mathrm{HH}}=2.2 \mathrm{~Hz}, 1 \mathrm{H}\right), 2.28\left(\mathrm{dd},{ }^{2} J_{\mathrm{HH}}\right.$ $=12.9 \mathrm{~Hz}$ and $\left.{ }^{3} J_{\mathrm{HH}}=9.1 \mathrm{~Hz}, 1 \mathrm{H}\right), 1.02-0.99(\mathrm{~m}, 1 \mathrm{H}), 0.79-0.71(\mathrm{~m}, 3 \mathrm{H}) .{ }^{13} \mathrm{C} \mathrm{NMR}\left(\mathrm{CDCl}_{3}\right)$ : $\delta$ 175.0, 141.0, 128.7, 127.6, 126.5, 121.2, 58.4, 52.8, 44.4, 41.4, 35.0, 23.7, 16.5, 9.9.

Minor diastereomer of 4ad: ${ }^{1} \mathrm{H}$ NMR $\left(\mathrm{CDCl}_{3}\right): \delta$ 7.37-7.26 (m, 5H), $3.69(\mathrm{~s}, 3 \mathrm{H}), 3.20$ $\left(\mathrm{ddd},{ }^{2} J_{\mathrm{HH}}=13.3 \mathrm{~Hz}\right.$ and ${ }^{3} J_{\mathrm{HH}}=6.1 \mathrm{~Hz}$ and $\left.{ }^{4} J_{\mathrm{HH}}=1.4 \mathrm{~Hz}, 1 \mathrm{H}\right), 3.02\left(\mathrm{dd},{ }^{3} J_{\mathrm{HH}}=9.3\right.$ and 6.2 $\mathrm{Hz}, 1 \mathrm{H}), 2.58\left(\mathrm{dd},{ }^{2} J_{\mathrm{HH}}=13.3 \mathrm{~Hz}\right.$ and $\left.{ }^{3} J_{\mathrm{HH}}=9.2 \mathrm{~Hz}, 1 \mathrm{H}\right), 2.57\left(\mathrm{dd},{ }^{2} J_{\mathrm{HH}}=12.9 \mathrm{~Hz}\right.$ and ${ }^{4} J_{\mathrm{HH}}=$ $1.2 \mathrm{~Hz}, 1 \mathrm{H}), 2.30\left(\mathrm{~d},{ }^{2} J_{\mathrm{HH}}=12.9 \mathrm{~Hz}, 1 \mathrm{H}\right), 1.06-1.02(\mathrm{~m}, 1 \mathrm{H}), 0.72-0.62(\mathrm{~m}, 3 \mathrm{H}) .{ }^{13} \mathrm{C} \mathrm{NMR}$ $\left(\mathrm{CDCl}_{3}\right): \delta 174.7,141.2,128.8,127.6,126.4,120.2,58.1,52.8,44.4,40.7,34.3,23.2,13.3$, 11.2.

Anal. Calcd for $\mathrm{C}_{16} \mathrm{H}_{17} \mathrm{NO}_{2}$ : C, 75.27; H, 6.71. Found: C, 75.11; H, 6.86.

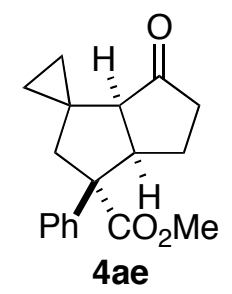

Entry 9. $89 \%$ yield $(\mathrm{dr}=65 / 35)$ along with $2 \%$ yield of 3ae. The relative configuration was determined by X-ray crystallographic analysis of the minor diastereomer.

Major diastereomer of 4ae: ${ }^{1} \mathrm{H}$ NMR $\left(\mathrm{CDCl}_{3}\right): \delta 7.35-7.25(\mathrm{~m}, 5 \mathrm{H}), 3.99\left(\mathrm{q},{ }^{3} J_{\mathrm{HH}}=8.9 \mathrm{~Hz}\right.$, $1 \mathrm{H}), 3.63(\mathrm{~s}, 3 \mathrm{H}), 2.52\left(\mathrm{~d},{ }^{2} J_{\mathrm{HH}}=12.5 \mathrm{~Hz}, 1 \mathrm{H}\right), 2.34\left(\mathrm{~d},{ }^{3} J_{\mathrm{HH}}=8.0 \mathrm{~Hz}, 1 \mathrm{H}\right), 2.26-2.13(\mathrm{~m}, 3 \mathrm{H})$, $1.82-1.75(\mathrm{~m}, 1 \mathrm{H}), 1.21-1.13(\mathrm{~m}, 2 \mathrm{H}), 0.58\left(\mathrm{ddd},{ }^{2} J_{\mathrm{HH}}=9.9 \mathrm{~Hz}\right.$ and ${ }^{3} J_{\mathrm{HH}}=6.4$ and $3.9 \mathrm{~Hz}$, $1 \mathrm{H}), 0.52-0.44(\mathrm{~m}, 2 \mathrm{H}) .{ }^{13} \mathrm{C} \mathrm{NMR}\left(\mathrm{CDCl}_{3}\right): \delta 219.9,176.0,139.3,128.6,127.5$ 127.4, 62.1, 55.5, 52.5, 48.5, 41.5, 38.9, 23.7, 23.5, 17.4, 5.3. Anal. Calcd for $\mathrm{C}_{18} \mathrm{H}_{20} \mathrm{O}_{3}: \mathrm{C}, 76.03 ; \mathrm{H}, 7.09$. Found: C, 75.80; H, 7.15.

Minor diastereomer of 4ae: ${ }^{1} \mathrm{H}$ NMR $\left(\mathrm{CDCl}_{3}\right): \delta 7.43\left(\mathrm{~d},{ }^{3} J_{\mathrm{HH}}=7.2 \mathrm{~Hz}, 2 \mathrm{H}\right), 7.35\left(\mathrm{t},{ }^{3} J_{\mathrm{HH}}\right.$ $=7.2 \mathrm{~Hz}, 2 \mathrm{H}), 7.28\left(\mathrm{tt},{ }^{3} J_{\mathrm{HH}}=7.3 \mathrm{~Hz}\right.$ and $\left.{ }^{4} J_{\mathrm{HH}}=1.2 \mathrm{~Hz}, 1 \mathrm{H}\right), 3.65(\mathrm{~s}, 3 \mathrm{H}), 3.56\left(\mathrm{q},{ }^{3} J_{\mathrm{HH}}=8.5\right.$ $\mathrm{Hz}, 1 \mathrm{H}), 2.82\left(\mathrm{~d},{ }^{2} J_{\mathrm{HH}}=13.7 \mathrm{~Hz}, 1 \mathrm{H}\right), 2.45-2.40(\mathrm{~m}, 1 \mathrm{H}), 2.36\left(\mathrm{~d},{ }^{3} J_{\mathrm{HH}}=7.7 \mathrm{~Hz}, 1 \mathrm{H}\right), 2.33-$ $2.22(\mathrm{~m}, 2 \mathrm{H}), 1.92\left(\mathrm{dd},{ }^{2} J_{\mathrm{HH}}=13.7 \mathrm{~Hz}\right.$ and $\left.{ }^{4} J_{\mathrm{HH}}=1.0 \mathrm{~Hz}, 1 \mathrm{H}\right), 1.81-1.73(\mathrm{~m}, 1 \mathrm{H}), 0.97-0.93$ 
$(\mathrm{m}, 1 \mathrm{H}), 0.36-0.29(\mathrm{~m}, 3 \mathrm{H}) .{ }^{13} \mathrm{C} \mathrm{NMR}\left(\mathrm{CDCl}_{3}\right): \delta 218.9,174.4,142.4,128.7,127.3,126.2$, 62.0, 55.8, 52.2, 50.1, 44.3, 38.8, 25.0, 22.1, 17.2, 6.8. Anal. Calcd for $\mathrm{C}_{18} \mathrm{H}_{20} \mathrm{O}_{3}$ : C, 76.03; $\mathrm{H}$, 7.09. Found: C, 75.88; H, 7.10.

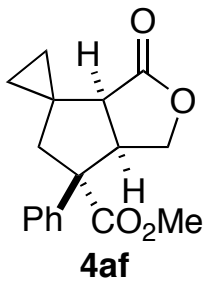

Entry 10. $94 \%$ yield $(\mathrm{dr}=57 / 43)$ along with trace amount of 3af. The relative configuration was assigned by analogy with 4ae (entry 9).

Major diastereomer of 4af: ${ }^{1} \mathrm{H}$ NMR $\left(\mathrm{CDCl}_{3}\right): \delta 7.33\left(\mathrm{t},{ }^{3} J_{\mathrm{HH}}=7.8 \mathrm{~Hz}, 2 \mathrm{H}\right), 7.29\left(\mathrm{tt},{ }^{3} J_{\mathrm{HH}}\right.$ $=7.7 \mathrm{~Hz}$ and $\left.{ }^{4} J_{\mathrm{HH}}=1.3 \mathrm{~Hz}, 1 \mathrm{H}\right), 7.23\left(\mathrm{~d},{ }^{3} J_{\mathrm{HH}}=8.3 \mathrm{~Hz}, 2 \mathrm{H}\right), 4.35\left(\mathrm{dddd},{ }^{3} J_{\mathrm{HH}}=10.0,8.8\right.$, and $7.3 \mathrm{~Hz}$ and $\left.{ }^{4} J_{\mathrm{HH}}=1.1 \mathrm{~Hz}, 1 \mathrm{H}\right), 4.17\left(\mathrm{t}, J_{\mathrm{HH}}=10.0 \mathrm{~Hz}, 1 \mathrm{H}\right), 3.65(\mathrm{~s}, 3 \mathrm{H}), 3.52\left(\mathrm{dd},{ }^{2} J_{\mathrm{HH}}=10.0\right.$ $\mathrm{Hz}$ and $\left.{ }^{3} J_{\mathrm{HH}}=7.1 \mathrm{~Hz}, 1 \mathrm{H}\right), 2.59\left(\mathrm{~d},{ }^{3} J_{\mathrm{HH}}=8.8 \mathrm{~Hz}, 1 \mathrm{H}\right), 2.54\left(\mathrm{~d},{ }^{2} J_{\mathrm{HH}}=12.7 \mathrm{~Hz}, 1 \mathrm{H}\right), 2.21(\mathrm{dd}$, ${ }^{2} J_{\mathrm{HH}}=12.8 \mathrm{~Hz}$ and $\left.{ }^{4} J_{\mathrm{HH}}=1.2 \mathrm{~Hz}, 1 \mathrm{H}\right), 1.31-1.24(\mathrm{~m}, 1 \mathrm{H}), 0.66-0.59(\mathrm{~m}, 3 \mathrm{H}) .{ }^{13} \mathrm{C} \mathrm{NMR}$ $\left(\mathrm{CDCl}_{3}\right): \delta 178.5,175.0,138.2,129.1,128.0127 .1,69.0,62.0,52.7,48.8,46.3,40.7,24.8$, 17.5, 4.8. Anal. Calcd for $\mathrm{C}_{17} \mathrm{H}_{18} \mathrm{O}_{4}$ : C, 71.31; H, 6.34. Found: C, 71.40; H, 6.59.

Minor diastereomer of 4af: ${ }^{1} \mathrm{H}$ NMR $\left(\mathrm{CDCl}_{3}\right): \delta 7.42\left(\mathrm{~d},{ }^{3} \mathrm{~J}_{\mathrm{HH}}=7.9 \mathrm{~Hz}, 2 \mathrm{H}\right), 7.38\left(\mathrm{t},{ }^{3} J_{\mathrm{HH}}\right.$ $=7.9 \mathrm{~Hz}, 2 \mathrm{H}), 7.32\left(\mathrm{tt},{ }^{3} J_{\mathrm{HH}}=7.4 \mathrm{~Hz}\right.$ and $\left.{ }^{4} J_{\mathrm{HH}}=1.4 \mathrm{~Hz}, 1 \mathrm{H}\right), 4.66\left(\mathrm{dd},{ }^{2} J_{\mathrm{HH}}=9.9 \mathrm{~Hz}\right.$ and ${ }^{3} J_{\mathrm{HH}}$ $=9.5 \mathrm{~Hz}, 1 \mathrm{H}), 4.07\left(\mathrm{dd},{ }^{2} J_{\mathrm{HH}}=10.0 \mathrm{~Hz}\right.$ and $\left.{ }^{3} J_{\mathrm{HH}}=8.1 \mathrm{~Hz}, 1 \mathrm{H}\right), 3.91\left(\mathrm{dddd},{ }^{3} J_{\mathrm{HH}}=9.4,8.4\right.$, and $8.2 \mathrm{~Hz}$ and $\left.{ }^{4} J_{\mathrm{HH}}=1.2 \mathrm{~Hz}, 1 \mathrm{H}\right), 3.65(\mathrm{~s}, 3 \mathrm{H}), 2.77\left(\mathrm{~d},{ }^{2} J_{\mathrm{HH}}=13.9 \mathrm{~Hz}, 1 \mathrm{H}\right), 2.58\left(\mathrm{~d},{ }^{3} J_{\mathrm{HH}}=\right.$ $8.4 \mathrm{~Hz}, 1 \mathrm{H}), 2.02\left(\mathrm{dd},{ }^{2} J_{\mathrm{HH}}=13.8 \mathrm{~Hz}\right.$ and $\left.{ }^{4} J_{\mathrm{HH}}=1.2 \mathrm{~Hz}, 1 \mathrm{H}\right), 1.13\left(\mathrm{ddd},{ }^{2} J_{\mathrm{HH}}=11.5 \mathrm{~Hz}\right.$ and ${ }^{3} J_{\mathrm{HH}}=6.5$ and $\left.5.3 \mathrm{~Hz}, 1 \mathrm{H}\right), 0.49\left(\mathrm{ddd},{ }^{2} J_{\mathrm{HH}}=11.7 \mathrm{~Hz}\right.$ and ${ }^{3} J_{\mathrm{HH}}=6.5$ and $\left.5.1 \mathrm{~Hz}, 1 \mathrm{H}\right), 0.46-$ $0.42(\mathrm{~m}, 1 \mathrm{H}), 0.35\left(\mathrm{ddd},{ }^{2} J_{\mathrm{HH}}=10.0 \mathrm{~Hz}\right.$ and ${ }^{3} J_{\mathrm{HH}}=6.0$ and $\left.4.4 \mathrm{~Hz}, 1 \mathrm{H}\right) .{ }^{13} \mathrm{C} \mathrm{NMR}\left(\mathrm{CDCl}_{3}\right): \delta$ 177.8, 173.7, 140.8, 129.0, 127.9, 126.1, 70.7, 61.8, 52.6, 48.7, 48.6, 43.4, 23.3, 18.5, 6.4. Anal. Calcd for $\mathrm{C}_{17} \mathrm{H}_{18} \mathrm{O}_{4}$ : C, 71.31; H, 6.34. Found: C, 71.24; H, 6.46.

\section{Typical Procedure for Equation 1.}

A solution of $\mathrm{PdCp}\left(\eta^{3}-\mathrm{C}_{3} \mathrm{H}_{5}\right)(3.2 \mathrm{mg}, 15 \mu \mathrm{mol}), \mathrm{P}(o-\mathrm{Tol})_{3}(9.1 \mathrm{mg}, 30 \mu \mathrm{mol}), 1 \mathrm{a}(133$ $\mathrm{mg}, 0.54 \mathrm{mmol})$, and $2 \mathbf{a}(27 \mu \mathrm{L}, 0.30 \mathrm{mmol})$ in 1,2-dichloroethane $(0.75 \mathrm{~mL})$ was stirred for $48 \mathrm{~h}$ at $60{ }^{\circ} \mathrm{C}$. The reaction mixture was directly passed through a pad of silica gel with EtOAc and the solvent was removed under vacuum. The residue was purified by silica gel preparative TLC with hexane/EtOAc/ $\mathrm{CH}_{2} \mathrm{Cl}_{2}=10 / 2 / 1$ to afford cycloadducts.

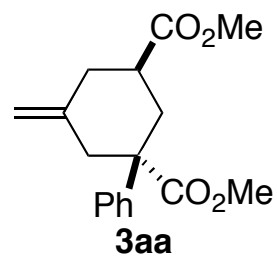

$83 \%$ yield $(\mathrm{dr}=71 / 29)$ along with $4 \%$ yield of $4 \mathbf{a a}$. The relative configuration was determined by X-ray crystallographic analysis of the major diastereomer after converting it to the corresponding monocarboxylic acid (S1).

Major diastereomer of 3aa: ${ }^{1} \mathrm{H}$ NMR $\left(\mathrm{CDCl}_{3}\right): \delta 7.38\left(\mathrm{~d},{ }^{3} J_{\mathrm{HH}}=7.2 \mathrm{~Hz}, 2 \mathrm{H}\right), 7.34\left(\mathrm{t},{ }^{3} J_{\mathrm{HH}}\right.$ $=7.2 \mathrm{~Hz}, 2 \mathrm{H}), 7.26\left(\mathrm{tt},{ }^{3} J_{\mathrm{HH}}=7.1 \mathrm{~Hz}\right.$ and $\left.{ }^{4} J_{\mathrm{HH}}=1.5 \mathrm{~Hz}, 1 \mathrm{H}\right), 4.89\left(\mathrm{q}, J_{\mathrm{HH}}=1.7 \mathrm{~Hz}, 1 \mathrm{H}\right), 4.88$ $\left(\mathrm{q}, J_{\mathrm{HH}}=1.6 \mathrm{~Hz}, 1 \mathrm{H}\right), 3.70(\mathrm{~s}, 3 \mathrm{H}), 3.63(\mathrm{~s}, 3 \mathrm{H}), 3.21\left(\mathrm{dt},{ }^{2} J_{\mathrm{HH}}=12.9 \mathrm{~Hz}\right.$ and ${ }^{4} J_{\mathrm{HH}}=2.0 \mathrm{~Hz}$, 
$1 \mathrm{H}), 2.91-2.87(\mathrm{~m}, 1 \mathrm{H}), 2.76\left(\mathrm{tt},{ }^{3} J_{\mathrm{HH}}=12.7\right.$ and $\left.4.0 \mathrm{~Hz}, 1 \mathrm{H}\right), 2.58\left(\mathrm{ddt},{ }^{2} J_{\mathrm{HH}}=13.2 \mathrm{~Hz}\right.$ and ${ }^{3} J_{\mathrm{HH}}=4.3 \mathrm{~Hz}$ and $\left.{ }^{4} J_{\mathrm{HH}}=1.3 \mathrm{~Hz}, 1 \mathrm{H}\right), 2.36\left(\mathrm{dq},{ }^{2} J_{\mathrm{HH}}=12.8 \mathrm{~Hz}\right.$ and $\left.{ }^{4} J_{\mathrm{HH}}=1.3 \mathrm{~Hz}, 1 \mathrm{H}\right), 2.17$ (tq, $J_{\mathrm{HH}}=12.9 \mathrm{~Hz}$ and $\left.{ }^{4} J_{\mathrm{HH}}=1.3 \mathrm{~Hz}, 1 \mathrm{H}\right), 1.75\left(\mathrm{dd},{ }^{2} J_{\mathrm{HH}}=13.4 \mathrm{~Hz}\right.$ and $\left.{ }^{3} J_{\mathrm{HH}}=12.6 \mathrm{~Hz}, 1 \mathrm{H}\right)$. ${ }^{13} \mathrm{C} \mathrm{NMR}\left(\mathrm{CDCl}_{3}\right): \delta 175.3,174.2,143.3,143.0,128.8,127.4,125.5,112.2,52.8,52.3,51.9$, 42.2, 41.9, 37.3, 36.7. Anal. Calcd for $\mathrm{C}_{17} \mathrm{H}_{20} \mathrm{O}_{4}: \mathrm{C}, 70.81 ; \mathrm{H}, 6.99$. Found: $\mathrm{C}, 71.06 ; \mathrm{H}, 7.00$.

Minor diastereomer of 3aa: ${ }^{1} \mathrm{H}$ NMR $\left(\mathrm{CDCl}_{3}\right): \delta 7.45\left(\mathrm{~d},{ }^{3} J_{\mathrm{HH}}=7.3 \mathrm{~Hz}, 2 \mathrm{H}\right), 7.33\left(\mathrm{t},{ }^{3} J_{\mathrm{HH}}\right.$ $=7.3 \mathrm{~Hz}, 2 \mathrm{H}), 7.23\left(\mathrm{tt},{ }^{3} J_{\mathrm{HH}}=7.3 \mathrm{~Hz}\right.$ and $\left.{ }^{4} J_{\mathrm{HH}}=1.1 \mathrm{~Hz}, 1 \mathrm{H}\right), 4.91\left(\mathrm{q}, J_{\mathrm{HH}}=1.8 \mathrm{~Hz}, 1 \mathrm{H}\right), 4.87$ $\left(\mathrm{q}, J_{\mathrm{HH}}=1.8 \mathrm{~Hz}, 1 \mathrm{H}\right), 3.68(\mathrm{~s}, 3 \mathrm{H}), 3.61(\mathrm{~s}, 3 \mathrm{H}), 3.33\left(\mathrm{dt},{ }^{2} J_{\mathrm{HH}}=14.4 \mathrm{~Hz}\right.$ and ${ }^{4} J_{\mathrm{HH}}=1.9 \mathrm{~Hz}$, $1 \mathrm{H}), 2.92\left(\mathrm{dq},{ }^{2} J_{\mathrm{HH}}=14.2 \mathrm{~Hz}\right.$ and $\left.{ }^{4} J_{\mathrm{HH}}=2.4 \mathrm{~Hz}, 1 \mathrm{H}\right), 2.52\left(\mathrm{dq},{ }^{2} J_{\mathrm{HH}}=14.4 \mathrm{~Hz}\right.$ and ${ }^{4} J_{\mathrm{HH}}=1.7$ $\mathrm{Hz}, 1 \mathrm{H}), 2.50-2.46(\mathrm{~m}, 1 \mathrm{H}), 2.31\left(\mathrm{tdd},{ }^{3} J_{\mathrm{HH}}=12.5,3.7\right.$, and $\left.3.1 \mathrm{~Hz}, 1 \mathrm{H}\right), 2.20\left(\mathrm{tq}, J_{\mathrm{HH}}=12.9\right.$ $\mathrm{Hz}$ and $\left.{ }^{4} J_{\mathrm{HH}}=1.5 \mathrm{~Hz}, 1 \mathrm{H}\right), 2.10\left(\mathrm{dd},{ }^{2} J_{\mathrm{HH}}=14.1 \mathrm{~Hz}\right.$ and $\left.{ }^{3} J_{\mathrm{HH}}=12.6 \mathrm{~Hz}, 1 \mathrm{H}\right),{ }^{13} \mathrm{C} \mathrm{NMR}$ $\left(\mathrm{CDCl}_{3}\right): \delta 175.6,175.3,142.2,139.3,128.7,127.7,127.1,113.4,52.6,51.9,50.4,40.1,38.9$, 36.6, 36.0. Anal. Calcd for $\mathrm{C}_{17} \mathrm{H}_{20} \mathrm{O}_{4}$ : C, 70.81; H, 6.99. Found: C, 71.01; H, 7.12.

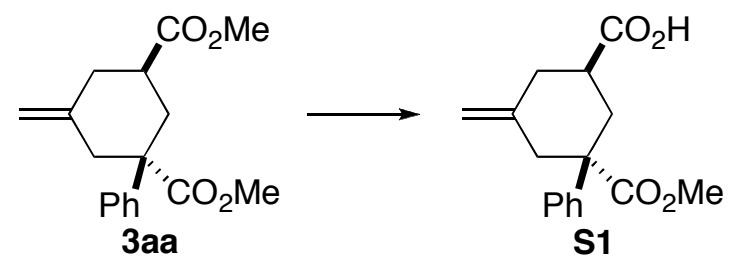

A solution of $\mathrm{KOH}(265 \mathrm{mg}, 4.01 \mathrm{mmol} ; 85 \%)$ in $\mathrm{MeOH} / \mathrm{H}_{2} \mathrm{O}(2.0 \mathrm{~mL} / 1.0 \mathrm{~mL})$ was added to a solution of 3aa (170 mg, $0.590 \mathrm{mmol}$; major diastereomer) in $\mathrm{MeOH}(7.0 \mathrm{~mL})$ and the mixture was refluxed for $12 \mathrm{~h}$. After cooled to room temperature, the solvent was concentrated under vacuum, and this was diluted with $\mathrm{H}_{2} \mathrm{O}(10 \mathrm{~mL})$. The mixture was washed with $\mathrm{Et}_{2} \mathrm{O}(20 \mathrm{~mL})$ and the aqueous layer was acidified with $6 \mathrm{~N} \mathrm{HClaq}$. This was then extracted with $\mathrm{Et}_{2} \mathrm{O}$ (20 mL x 7 times), and the organic layer was dried over $\mathrm{MgSO}_{4}$, filtered, and concentrated under vacuum. The residue was purified by silica gel preparative TLC with hexane/EtOAc $=1 / 1$ to afford $\mathbf{S 1}$ as a pale yellow solid $(114 \mathrm{mg}, 0.416 \mathrm{mmol} ; 70 \%$ yield).

${ }^{1} \mathrm{H}$ NMR $\left(\mathrm{CDCl}_{3}\right): \delta 7.39-7.32(\mathrm{~m}, 4 \mathrm{H}), 7.27\left(\mathrm{tt},{ }^{3} J_{\mathrm{HH}}=7.1 \mathrm{~Hz}\right.$ and $\left.{ }^{4} J_{\mathrm{HH}}=1.5 \mathrm{~Hz}, 1 \mathrm{H}\right)$, 4.92-4.89 (m, 2H), $3.63(\mathrm{~s}, 3 \mathrm{H}), 3.23\left(\mathrm{dt},{ }^{2} J_{\mathrm{HH}}=12.9 \mathrm{~Hz}\right.$ and $\left.{ }^{4} J_{\mathrm{HH}}=1.8 \mathrm{~Hz}, 1 \mathrm{H}\right), 2.95-2.90(\mathrm{~m}$, $1 \mathrm{H}), 2.81\left(\mathrm{tt},{ }^{3} J_{\mathrm{HH}}=12.7\right.$ and $\left.3.9 \mathrm{~Hz}, 1 \mathrm{H}\right), 2.66-2.61(\mathrm{~m}, 1 \mathrm{H}), 2.37\left(\mathrm{~d},{ }^{2} J_{\mathrm{HH}}=12.9 \mathrm{~Hz}, 1 \mathrm{H}\right)$, $2.18\left(\mathrm{t}, J_{\mathrm{HH}}=13.1 \mathrm{~Hz}, 1 \mathrm{H}\right), 1.76\left(\mathrm{t}, J_{\mathrm{HH}}=13.0 \mathrm{~Hz}, 1 \mathrm{H}\right) .{ }^{13} \mathrm{C} \mathrm{NMR}\left(\mathrm{CDCl}_{3}\right): \delta 180.3,174.2$, 142.93, 142.87, 128.9, 127.5, 125.6, 112.5, 52.8, 52.4, 42.2, 41.7, 37.1, 36.5. Anal. Calcd for $\mathrm{C}_{16} \mathrm{H}_{18} \mathrm{O}_{4}$ : C, 70.06; H, 6.61. Found: C, 69.82; H, 6.69. 


\section{X-ray Crystal Structure of Major Diastereomer of Compound 4ea}

\section{Data Collection}

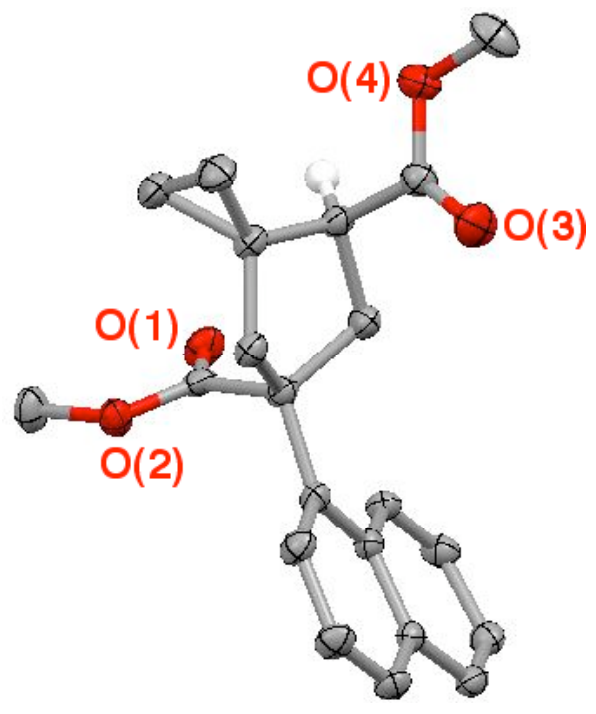

A colorless $\mathrm{Et}_{2} \mathrm{O}$ solution of compound 4ea was prepared. Crystals suitable for X-ray analysis were obtained by slow evaporation of the solvent.

A colorless prism crystal of $\mathrm{C}_{21} \mathrm{H}_{22} \mathrm{O}_{4}$ having approximate dimensions of $0.50 \times 0.40 \mathrm{x}$ $0.40 \mathrm{~mm}$ was mounted on a glass fiber. All measurements were made on a Rigaku RAXIS RAPID imaging plate area detector with graphite monochromated Mo-K $\alpha$ radiation.

Indexing was performed from 3 oscillations that were exposed for 60 seconds. The crystal-to-detector distance was $127.40 \mathrm{~mm}$.

Cell constants and an orientation matrix for data collection corresponded to a C-centered monoclinic cell with dimensions:

$$
\begin{aligned}
& \mathrm{a}=24.204(11) \AA \\
& \mathrm{b}=11.794(5) \AA \quad \AA=131.987(16)^{\circ} \\
& \mathrm{c}=16.099(7) \AA \\
& \mathrm{V}=3416.0(26) \AA^{3}
\end{aligned}
$$

For $\mathrm{Z}=6$ and F.W. $=338.40$, the calculated density is $0.987 \mathrm{~g} / \mathrm{cm}^{3}$. Based on the systematic absences of:

hkl: $\mathrm{h}+\mathrm{k} \pm 2 \mathrm{n}$

h0l: $1 \pm 2 n$

packing considerations, a statistical analysis of intensity distribution, and the successful solution and refinement of the structure, the space group was determined to be:

\section{$\mathrm{C} 2 / \mathrm{c}(\# 15)$}

The data were collected at a temperature of $-150 \pm 1{ }^{\circ} \mathrm{C}$ to a maximum $2 \theta$ value of $55.0^{\circ}$. A total of 44 oscillation images were collected. A sweep of data was done using $\omega$ scans from 130.0 to $190.0^{\circ}$ in $5.0^{\circ}$ step, at $\chi=45.0^{\circ}$ and $\phi=0.0^{\circ}$. The exposure rate was $60.0\left[\mathrm{sec} . /^{\circ}\right.$ ]. A second sweep was performed using $\omega$ scans from 0.0 to $160.0^{\circ}$ in $5.0^{\circ} \mathrm{step}$, at $\chi=45.0^{\circ}$ and $\phi=180.0^{\circ}$. The exposure rate was 60.0 [sec. $/^{\circ}$ ]. The crystal-to-detector distance was 127.40 $\mathrm{mm}$. Readout was performed in the $0.100 \mathrm{~mm}$ pixel mode. 


\section{Data Reduction}

Of the 16311 reflections that were collected, 3916 were unique $(\operatorname{Rint}=0.038)$.

The linear absorption coefficient, $\mu$, for Mo-K $\alpha$ radiation is $0.676 \mathrm{~cm}^{-1}$. The data were corrected for Lorentz and polarization effects.

\section{Structure Solution and Refinement}

The structure was solved by direct methods ${ }^{4}$ and expanded using Fourier techniques. ${ }^{5}$ The non-hydrogen atoms were refined anisotropically. Hydrogen atoms were refined isotopically. The final cycle of full-matrix least-squares refinement ${ }^{6}$ on $\mathrm{F}$ was based on 11643 observed reflections $(\mathrm{I}>2.00 \sigma(\mathrm{I}))$ and 314 variable parameters and converged (largest parameter shift was 0.00 times its esd) with unweighted and weighted agreement factors of:

$$
\begin{gathered}
\mathrm{R}=\Sigma\|\mathrm{Fo}|-| \mathrm{Fc}\| / \Sigma|\mathrm{Fo}|=0.0469 \\
\mathrm{R}_{\mathrm{W}}=\left[\Sigma \mathrm{w}(|\mathrm{Fo}|-|\mathrm{Fc}|)^{2} / \Sigma \mathrm{w} \mathrm{Fo}^{2}\right]^{1 / 2}=0.0552
\end{gathered}
$$

The standard deviation of an observation of unit weight ${ }^{7}$ was 0.86 . A Sheldrick weighting scheme was used. Plots of $\Sigma \mathrm{w}(|\mathrm{Fo}|-\mid \mathrm{Fcl})^{2}$ versus $\mid \mathrm{Fol}$, reflection order in data collection, sin $\theta / \lambda$ and various classes of indices showed no unusual trends. The maximum and minimum peaks on the final difference Fourier map corresponded to 0.98 and $-0.73 \mathrm{e}^{-} / \AA^{3}$, respectively.

Neutral atom scattering factors were taken from Cromer and Waber. ${ }^{8}$ Anomalous dispersion effects were included in Fcalc; ${ }^{9}$ the values for $\Delta f^{\prime}$ and $\Delta f^{\prime \prime}$ were those of Creagh and McAuley. ${ }^{10}$ The values for the mass attenuation coefficients are those of Creagh and Hubbell. ${ }^{11}$ All calculations were performed using the CrystalStructure ${ }^{12,13}$ crystallographic software package.

${ }^{4}$ SIR92: Altomare, A.; Cascarano, G.; Giacovazzo, C.; Guagliardi, A.; Burla, M.; Polidori, G.; Camalli, M. J. Appl. Cryst. 1994, 27, 435.

${ }^{5}$ DIRDIF99: Beurskens, P. T.; Admiraal, G.; Beurskens, G.; Bosman, W. P.; de Gelder, R.; Israel, R.; Smits, J. M. M. The DIRDIF-99 program system, Technical Report of the Crystallography Laboratory, University of Nijmegen, The Netherlands (1999).

${ }^{6}$ Least Squares function minimized:

$$
\Sigma w\left(\left|\mathrm{~F}_{\mathrm{O}}\right|-\left|\mathrm{F}_{\mathrm{C}}\right|\right)^{2} \quad \text { where } \mathrm{w}=\text { Least Squares weights. }
$$

${ }^{7}$ Standard deviation of an observation of unit weight:

$$
\begin{aligned}
& {\left[\Sigma w\left(\left|\mathrm{~F}_{\mathrm{O}}\right|-\left|\mathrm{F}_{\mathrm{C}}\right|\right)^{2} /\left(\mathrm{N}_{\mathrm{O}}-\mathrm{N}_{\mathrm{V}}\right)\right]^{1 / 2}} \\
& \text { where: } \mathrm{N}_{\mathrm{O}}=\text { number of observations, } \mathrm{N}_{\mathrm{V}}=\text { number of variables }
\end{aligned}
$$

${ }^{8}$ Cromer, D. T.; Waber, J. T. "International Tables for X-ray Crystallography", Vol. IV, The Kynoch Press, Birmingham, England, Table 2.2 A (1974).

${ }^{9}$ Ibers, J. A.; Hamilton, W. C. Acta Crystallogr. 1964, 17, 781.

${ }^{10}$ Creagh, D. C.; McAuley, W. J. "International Tables for Crystallography", Vol C, (Wilson, A. J. C. ed.), Kluwer Academic Publishers, Boston, Table 4.2.6.8, pages 219-222 (1992).

${ }^{11}$ Creagh, D. C.; Hubbell, J. H. "International Tables for Crystallography", Vol C, (Wilson, A. J. C. ed.), Kluwer Academic Publishers, Boston, Table 4.2.4.3, pages 200-206 (1992).

${ }^{12}$ CrystalStructure 3.8.0: Crystal Structure Analysis Package, Rigaku and Rigaku/MSC (2000-2006). 9009 New Trails Dr. The Woodlands TX 77381 USA.

${ }^{13}$ CRYSTALS Issue 11: Carruthers, J. R.; Rollett, J. S.; Betteridge, P. W.; Kinna, D.; Pearce, L.; Larsen, A.; Gabe, E. Chemical Crystallography Laboratory, Oxford, UK. (1999). 
The crystal structure has been deposited at the Cambridge Crystallographic Data Centre (deposition number: CCDC 659648). The data can be obtained free of charge via the Internet at www.ccdc.cam.ac.uk/conts/retrieving.html. 


\section{Experimental Details}

\section{A. Crystal Data}

Empirical Formula

Formula Weight

Crystal Color, Habit

Crystal Dimensions

Crystal System

Lattice Type

Indexing Images

Detector Position

Pixel Size

Lattice Parameters

Space Group

$\mathrm{Z}$ value

$\mathrm{D}_{\text {calc }}$

F000

$\mu(\operatorname{MoK} \alpha)$
$\mathrm{C}_{21} \mathrm{H}_{22} \mathrm{O}_{4}$

338.40

colorless, prism

$0.50 \times 0.40 \times 0.40 \mathrm{~mm}$

monoclinic

C-centered

3 oscillations @60.0 seconds

$127.40 \mathrm{~mm}$

$0.100 \mathrm{~mm}$

$$
\begin{aligned}
& \mathrm{a}=24.204(11) \AA \\
& \mathrm{b}=11.794(5) \AA \\
& \mathrm{c}=16.099(7) \AA \\
& \beta=131.987(16){ }^{\circ} \\
& \mathrm{V}=3416.0(26) \AA^{3}
\end{aligned}
$$

$\mathrm{C} 2 / \mathrm{c}(\# 15)$

6

$0.987 \mathrm{~g} / \mathrm{cm}^{3}$

1080.00

$0.676 \mathrm{~cm}^{-1}$ 


\section{B. Intensity Measurements}

Diffractometer

Radiation

Detector Aperture

Data Images

$\omega$ oscillation Range $(\chi=45.0, \phi=0.0)$

Exposure Rate

$\omega$ oscillation Range $(\chi=45.0, \phi=180.0)$

Exposure Rate

Detector Position

Pixel Size

$2 \theta_{\max }$

No. of Reflections Measured

Corrections
Rigaku RAXIS-RAPID

$\operatorname{MoK} \alpha(\lambda=0.71075 \AA)$

graphite monochromated

$280 \mathrm{~mm} \times 256 \mathrm{~mm}$

44 exposures

$130.0-190.0^{\circ}$

$60.0 \mathrm{sec} . /^{\circ}$

$0.0-160.0^{\circ}$

$60.0 \mathrm{sec} . /^{\circ}$

$127.40 \mathrm{~mm}$

$0.100 \mathrm{~mm}$

$55.0^{\circ}$

Total: 16311

Unique: $3916\left(\mathrm{R}_{\text {int }}=0.038\right)$

Lorentz-polarization 


\section{Structure Solution and Refinement}

Structure Solution

Refinement

Function Minimized

Least Squares Weights

$2 \theta_{\max }$ cutoff

Anomalous Dispersion

No. Observations $(\mathrm{I}>2.00 \sigma(\mathrm{I}))$

No. Variables

Reflection/Parameter Ratio

Residuals: R (I>2.00б(I))

Residuals: Rw (I>2.00\%(I))

Goodness of Fit Indicator

Max Shift/Error in Final Cycle

Maximum peak in Final Diff. Map

Minimum peak in Final Diff. Map
Direct Methods (SIR92)

Full-matrix least-squares on $\mathrm{F}$

$\Sigma \mathrm{w}(|\mathrm{Fol}-| \mathrm{Fcl})^{2}$

$1 /\left[0.0010 \mathrm{Fo}^{2}+3.0000 \sigma\left(\mathrm{Fo}^{2}\right)+0.5000\right]$

$55.0^{\circ}$

All non-hydrogen atoms

11643

314

37.08

0.0469

0.0552

0.858

0.000

$0.98 \mathrm{e}^{-} / \AA^{3}$

$-0.73 \mathrm{e}^{-} / \AA^{3}$ 


\section{X-ray Crystal Structure of Minor Diastereomer of Compound 4ae}

\section{Data Collection}

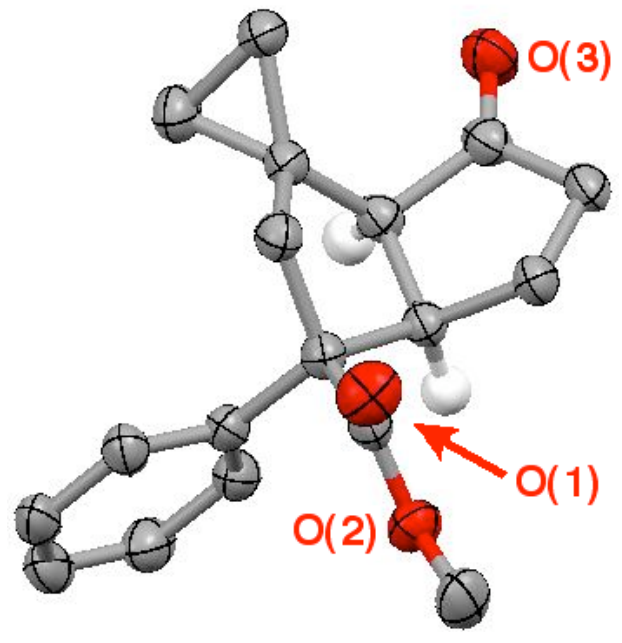

A colorless $\mathrm{Et}_{2} \mathrm{O}$ solution of compound 4ae was prepared. Crystals suitable for X-ray analysis were obtained by slow evaporation of the solvent.

A colorless prism crystal of $\mathrm{C}_{18} \mathrm{H}_{20} \mathrm{O}_{3}$ having approximate dimensions of $0.50 \times 0.30 \mathrm{x}$ $0.05 \mathrm{~mm}$ was mounted on a glass fiber. All measurements were made on a Rigaku RAXIS RAPID imaging plate area detector with graphite monochromated Mo-K $\alpha$ radiation.

Indexing was performed from 3 oscillations that were exposed for 2000 seconds. The crystal-to-detector distance was $127.40 \mathrm{~mm}$.

Cell constants and an orientation matrix for data collection corresponded to a primitive orthorhombic cell with dimensions:

$$
\begin{aligned}
& \mathrm{a}=25.14(4) \AA \\
& \mathrm{b}=7.838(19) \AA \\
& \mathrm{c}=15.20(3) \AA \\
& \mathrm{V}=2995.9(105) \AA^{3}
\end{aligned}
$$

For $\mathrm{Z}=8$ and F.W. $=284.53$, the calculated density is $1.261 \mathrm{~g} / \mathrm{cm}^{3}$. The systematic absences of:

$$
\begin{aligned}
& \text { 0kl: } k \pm 2 n \\
& \text { h0l: } 1 \pm 2 n \\
& \text { hk0: } h+k \pm 2 n
\end{aligned}
$$

uniquely determine the space group to be:

\section{$\operatorname{Pbcn}(\# 60)$}

The data were collected at a temperature of $-150 \pm 1{ }^{\circ} \mathrm{C}$ to a maximum $2 \theta$ value of $55.0^{\circ}$. A total of 44 oscillation images were collected. A sweep of data was done using $\omega$ scans from 130.0 to $190.0^{\circ}$ in $5.0^{\circ}$ step, at $\chi=45.0^{\circ}$ and $\phi=0.0^{\circ}$. The exposure rate was $400.0\left[\mathrm{sec} . /^{\circ}\right.$ ]. A second sweep was performed using $\omega$ scans from 0.0 to $160.0^{\circ}$ in $5.0^{\circ}$ step, at $\chi=45.0^{\circ}$ and $\phi=180.0^{\circ}$. The exposure rate was 400.0 [sec. $/^{\circ}$ ]. The crystal-to-detector distance was $127.40 \mathrm{~mm}$. Readout was performed in the $0.100 \mathrm{~mm}$ pixel mode. 


\section{Data Reduction}

Of the 25285 reflections that were collected, 3370 were unique $\left(\mathrm{R}_{\mathrm{int}}=0.106\right)$.

The linear absorption coefficient, $\mu$, for Mo-K $\alpha$ radiation is $0.845 \mathrm{~cm}^{-1}$. The data were corrected for Lorentz and polarization effects.

\section{Structure Solution and Refinement}

The structure was solved by direct methods ${ }^{14}$ and expanded using Fourier techniques. ${ }^{5}$ The non-hydrogen atoms were refined anisotropically. Hydrogen atoms were refined isotopically. The final cycle of full-matrix least-squares refinement ${ }^{15}$ on $\mathrm{F}^{2}$ was based on 3370 observed reflections and 271 variable parameters and converged (largest parameter shift was 0.00 times its esd) with unweighted and weighted agreement factors of:

$$
\begin{gathered}
\mathrm{R} 1=\Sigma\|\mathrm{Fo}|-| \mathrm{Fc}\| / \Sigma|\mathrm{Fo}|=0.0641 \\
\mathrm{wR} 2=\left[\Sigma \mathrm{w}\left(\mathrm{Fo}^{2}-\mathrm{Fc}^{2}\right)^{2} / \Sigma \mathrm{w}\left(\mathrm{Fo}^{2}\right)^{2}\right]^{1 / 2}=0.1848
\end{gathered}
$$

The standard deviation of an observation of unit weight ${ }^{16}$ was 1.07 . Unit weights were used. The maximum and minimum peaks on the final difference Fourier map corresponded to 0.20 and $-0.29 \mathrm{e}^{-} / \AA^{3}$, respectively.

Neutral atom scattering factors were taken from Cromer and Waber. ${ }^{8}$ Anomalous dispersion effects were included in Fcalc; ${ }^{9}$ the values for $\Delta \mathrm{f}^{\prime}$ and $\Delta \mathrm{f}^{\prime \prime}$ were those of Creagh and McAuley. ${ }^{10}$ The values for the mass attenuation coefficients are those of Creagh and Hubbell. ${ }^{11}$ All calculations were performed using the CrystalStructure ${ }^{12}$ crystallographic software package except for refinement, which was performed using SHELXL-97. ${ }^{17}$

The crystal structure has been deposited at the Cambridge Crystallographic Data Centre (deposition number: CCDC 659649). The data can be obtained free of charge via the Internet at www.ccdc.cam.ac.uk/conts/retrieving.html.

${ }^{14}$ SIR97: Altomare, A.; Burla, M.; Camalli, M.; Cascarano, G.; Giacovazzo, C.; Guagliardi, A.; Moliterni, A.; Polidori, G.; Spagna, R. J. Appl. Cryst. 1999, 32, 115.

${ }^{15}$ Least Squares function minimized: (SHELXL97)

$$
\Sigma w\left(\mathrm{~F}_{\mathrm{O}}{ }^{2}-\mathrm{F}_{\mathrm{C}}{ }^{2}\right)^{2} \quad \text { where } \mathrm{w}=\text { Least Squares weights. }
$$

${ }^{16}$ Standard deviation of an observation of unit weight:

$$
\left[\Sigma w\left(\mathrm{~F}_{\mathrm{O}}{ }^{2}-\mathrm{F}_{\mathrm{C}}{ }^{2}\right)^{2} /\left(\mathrm{N}_{\mathrm{O}}-\mathrm{N}_{\mathrm{V}}\right)\right]^{1 / 2}
$$

where: $\mathrm{N}_{\mathrm{O}}=$ number of observations, $\mathrm{N}_{\mathrm{V}}=$ number of variables

${ }^{17}$ SHELX97: Sheldrick, G. M. (1997). 


\section{Experimental Details}

\section{A. Crystal Data}

Empirical Formula

Formula Weight

Crystal Color, Habit

Crystal Dimensions

Crystal System

Lattice Type

Indexing Images

Detector Position

Pixel Size

Lattice Parameters

Space Group

$\mathrm{Z}$ value

$D_{\text {calc }}$

F000

$\mu(\operatorname{MoK} \alpha)$
$\mathrm{C}_{18} \mathrm{H}_{20} \mathrm{O}_{3}$

284.35

colorless, prism

$0.50 \times 0.30 \times 0.05 \mathrm{~mm}$

orthorhombic

Primitive

3 oscillations@1999.8 seconds

$127.40 \mathrm{~mm}$

$0.100 \mathrm{~mm}$

$$
\begin{aligned}
& \mathrm{a}=25.14(4) \AA \\
& \mathrm{b}=7.838(19) \AA \\
& \mathrm{c}=15.20(3) \AA \\
& \mathrm{V}=2995.9(105) \AA^{3}
\end{aligned}
$$

Pbcn (\#60)

8

$1.261 \mathrm{~g} / \mathrm{cm}^{3}$

1216.00

$0.845 \mathrm{~cm}^{-1}$ 


\section{B. Intensity Measurements}

Diffractometer

Radiation

Detector Aperture

Data Images

$\omega$ oscillation Range $(\chi=45.0, \phi=0.0)$

Exposure Rate

$\omega$ oscillation Range $(\chi=45.0, \phi=180.0)$

Exposure Rate

Detector Position

Pixel Size

$2 \theta_{\max }$

No. of Reflections Measured

Corrections
Rigaku RAXIS-RAPID

$\operatorname{MoK} \alpha(\lambda=0.71075 \AA)$

graphite monochromated

$280 \mathrm{~mm} \times 256 \mathrm{~mm}$

44 exposures

$130.0-190.0^{\circ}$

$400.0 \mathrm{sec} . /^{\circ}$

$0.0-160.0^{\circ}$

$400.0 \mathrm{sec} . /^{\circ}$

$127.40 \mathrm{~mm}$

$0.100 \mathrm{~mm}$

$55.0^{\circ}$

Total: 25285

Unique: $3370\left(\mathrm{R}_{\text {int }}=0.106\right)$

Lorentz-polarization 


\section{Structure Solution and Refinement}

Structure Solution

Refinement

Function Minimized

Least Squares Weights

$2 \theta_{\max }$ cutoff

Anomalous Dispersion

No. Observations (All reflections)

No. Variables

Reflection/Parameter Ratio

Residuals: R1 (I>2.00\%(I))

Residuals: R (All reflections)

Residuals: wR2 (All reflections)

Goodness of Fit Indicator

Max Shift/Error in Final Cycle

Maximum peak in Final Diff. Map

Minimum peak in Final Diff. Map
Direct Methods (SIR97)

Full-matrix least-squares on $\mathrm{F}^{2}$

$\Sigma \mathrm{w}\left(\mathrm{Fo}^{2}-\mathrm{Fc}^{2}\right)^{2}$

$\mathrm{w}=1 /\left[\sigma^{2}\left(\mathrm{Fo}^{2}\right)+(0.1000 \cdot \mathrm{P})^{2}\right.$

$+0.0000 \bullet \mathrm{P}]$

where $\mathrm{P}=\left(\operatorname{Max}\left(\mathrm{Fo}^{2}, 0\right)+2 \mathrm{Fc}^{2}\right) / 3$

$55.0^{\circ}$

All non-hydrogen atoms

3370

271

12.44

0.0641

0.0969

0.1848

1.066

0.000

$0.20 \mathrm{e}^{-/ \AA^{3}}$

$-0.29 \mathrm{e}^{-} / \AA^{3}$ 


\section{X-ray Crystal Structure of Compound S1}

\section{Data Collection}

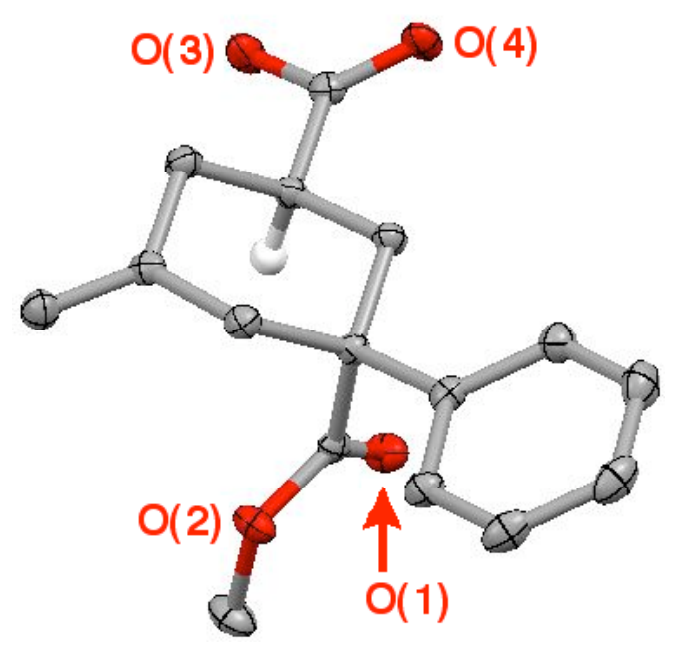

A colorless $\mathrm{Et}_{2} \mathrm{O}$ solution of compound $\mathbf{S 1}$ was prepared. Crystals suitable for X-ray analysis were obtained by slow evaporation of the solvent.

A colorless prism crystal of $\mathrm{C}_{16} \mathrm{H}_{18} \mathrm{O}_{4}$ having approximate dimensions of $0.50 \times 0.40 \mathrm{x}$ $0.30 \mathrm{~mm}$ was mounted on a glass fiber. All measurements were made on a Rigaku RAXIS RAPID imaging plate area detector with graphite monochromated Mo-K $\alpha$ radiation.

Indexing was performed from 3 oscillations that were exposed for 30 seconds. The crystal-to-detector distance was $127.40 \mathrm{~mm}$.

Cell constants and an orientation matrix for data collection corresponded to a primitive triclinic cell with dimensions:

$$
\begin{array}{ll}
\mathrm{a}=7.328(5) \AA & \alpha=72.48(3)^{\circ} \\
\mathrm{b}=8.183(5) \AA & \beta=88.50(3)^{\circ} \\
\mathrm{c}=12.226(10) \AA & \gamma=76.67(2)^{\circ} \\
\mathrm{V}=679.6(8) \AA^{3} &
\end{array}
$$

For $\mathrm{Z}=2$ and F.W. $=274.32$, the calculated density is $1.341 \mathrm{~g} / \mathrm{cm}^{3}$. Based on the statistical analysis of intensity distribution, and the successful solution and refinement of the structure, the space group was determined to be:

$$
\mathrm{P}-1(\# 2)
$$

The data were collected at a temperature of $-150 \pm 1{ }^{\circ} \mathrm{C}$ to a maximum $2 \theta$ value of $55.0^{\circ}$. A total of 44 oscillation images were collected. A sweep of data was done using $\omega$ scans from 130.0 to $190.0^{\circ}$ in $5.0^{\circ}$ step, at $\chi=45.0^{\circ}$ and $\phi=0.0^{\circ}$. The exposure rate was $60.0\left[\mathrm{sec} . /^{\circ}\right.$ ]. A second sweep was performed using $\omega$ scans from 0.0 to $160.0^{\circ}$ in $5.0^{\circ} \mathrm{step}$, at $\chi=45.0^{\circ}$ and $\phi=180.0^{\circ}$. The exposure rate was $60.0\left[\mathrm{sec} . /^{\circ}\right.$ ]. The crystal-to-detector distance was 127.40 $\mathrm{mm}$. Readout was performed in the $0.100 \mathrm{~mm}$ pixel mode.

\section{Data Reduction}

Of the 6644 reflections that were collected, 3071 were unique $\left(R_{i n t}=0.026\right)$.

The linear absorption coefficient, $\mu$, for Mo-K $\alpha$ radiation is $0.956 \mathrm{~cm}^{-1}$. The data were corrected for Lorentz and polarization effects. 


\section{Structure Solution and Refinement}

The structure was solved by direct methods ${ }^{14}$ and expanded using Fourier techniques. ${ }^{5}$ The non-hydrogen atoms were refined anisotropically. Hydrogen atoms were refined using the riding model. The final cycle of full-matrix least-squares refinement ${ }^{15}$ on $\mathrm{F}^{2}$ was based on 3071 observed reflections and 182 variable parameters and converged (largest parameter shift was 0.00 times its esd) with unweighted and weighted agreement factors of:

$$
\begin{gathered}
\mathrm{R} 1=\Sigma\|\mathrm{Fo}|-| \mathrm{Fc}\| / \Sigma|\mathrm{Fo}|=0.0437 \\
\mathrm{wR} 2=\left[\Sigma \mathrm{w}\left(\mathrm{Fo}^{2}-\mathrm{Fc}^{2}\right)^{2} / \Sigma \mathrm{w}\left(\mathrm{Fo}^{2}\right)^{2}\right]^{1 / 2}=0.1366
\end{gathered}
$$

The standard deviation of an observation of unit weight ${ }^{16}$ was 1.13 . Unit weights were used. The maximum and minimum peaks on the final difference Fourier map corresponded to 0.63 and $-0.65 \mathrm{e}^{-} / \AA^{3}$, respectively.

Neutral atom scattering factors were taken from Cromer and Waber. ${ }^{8}$ Anomalous dispersion effects were included in Fcalc; ${ }^{9}$ the values for $\Delta \mathrm{f}^{\prime}$ and $\Delta \mathrm{f}^{\prime \prime}$ were those of Creagh and McAuley. ${ }^{10}$ The values for the mass attenuation coefficients are those of Creagh and Hubbell. ${ }^{11}$ All calculations were performed using the CrystalStructure ${ }^{12}$ crystallographic software package except for refinement, which was performed using SHELXL-97. ${ }^{17}$

The crystal structure has been deposited at the Cambridge Crystallographic Data Centre (deposition number: CCDC 659650). The data can be obtained free of charge via the Internet at www.ccdc.cam.ac.uk/conts/retrieving.html. 


\section{Experimental Details}

\section{A. Crystal Data}

Empirical Formula

Formula Weight

Crystal Color, Habit

Crystal Dimensions

Crystal System

Lattice Type

Indexing Images

Detector Position

Pixel Size

Lattice Parameters

Space Group

$\mathrm{Z}$ value

$\mathrm{D}_{\text {calc }}$

F000

$\mu(\operatorname{MoK} \alpha)$
$\mathrm{C}_{16} \mathrm{H}_{18} \mathrm{O}_{4}$

274.32

colorless, prism

$0.50 \times 0.40 \times 0.30 \mathrm{~mm}$

triclinic

Primitive

3 oscillations@ 30.0 seconds

$127.40 \mathrm{~mm}$

$0.100 \mathrm{~mm}$

$$
\begin{aligned}
& \mathrm{a}=7.328(5) \AA \\
& \mathrm{b}=8.183(5) \AA \\
& \mathrm{c}=12.226(10) \AA \\
& \alpha=72.48(3)^{\circ} \\
& \beta=88.50(3)^{\circ} \\
& \gamma=76.67(2)^{\circ} \\
& \mathrm{V}=679.6(8) \AA^{3}
\end{aligned}
$$

$\mathrm{P}-1(\# 2)$

2

$1.341 \mathrm{~g} / \mathrm{cm}^{3}$

292.00

$0.956 \mathrm{~cm}^{-1}$ 


\section{B. Intensity Measurements}

Diffractometer

Radiation

Detector Aperture

Data Images

$\omega$ oscillation Range $(\chi=45.0, \phi=0.0)$

Exposure Rate

$\omega$ oscillation Range $(\chi=45.0, \phi=180.0)$

Exposure Rate

Detector Position

Pixel Size

$2 \theta_{\max }$

No. of Reflections Measured

Corrections
Rigaku RAXIS-RAPID

$\operatorname{MoK} \alpha(\lambda=0.71075 \AA)$

graphite monochromated

$280 \mathrm{~mm} \times 256 \mathrm{~mm}$

44 exposures

$130.0-190.0^{\circ}$

$60.0 \mathrm{sec} . /^{\circ}$

$0.0-160.0^{\circ}$

$60.0 \mathrm{sec} . /^{\circ}$

$127.40 \mathrm{~mm}$

$0.100 \mathrm{~mm}$

$55.0^{\circ}$

Total: 6644

Unique: $3071\left(\mathrm{R}_{\text {int }}=0.026\right)$

Lorentz-polarization 


\section{Structure Solution and Refinement}

Structure Solution

Refinement

Function Minimized

Least Squares Weights

$2 \theta_{\max }$ cutoff

Anomalous Dispersion

No. Observations (All reflections)

No. Variables

Reflection/Parameter Ratio

Residuals: R1 (I>2.00\%(I))

Residuals: R (All reflections)

Residuals: wR2 (All reflections)

Goodness of Fit Indicator

Max Shift/Error in Final Cycle

Maximum peak in Final Diff. Map

Minimum peak in Final Diff. Map
Direct Methods (SIR97)

Full-matrix least-squares on $\mathrm{F}^{2}$

$\Sigma \mathrm{w}\left(\mathrm{Fo}^{2}-\mathrm{Fc}^{2}\right)^{2}$

$\mathrm{w}=1 /\left[\sigma^{2}\left(\mathrm{Fo}^{2}\right)+(0.0670 \cdot \mathrm{P})^{2}\right.$

$+0.2630 \bullet \mathrm{P}]$

where $\mathrm{P}=\left(\operatorname{Max}\left(\mathrm{Fo}^{2}, 0\right)+2 \mathrm{Fc}^{2}\right) / 3$

$55.0^{\circ}$

All non-hydrogen atoms

3071

182

16.87

0.0437

0.0528

0.1366

1.129

0.000

$0.63 \mathrm{e}^{-/ \AA^{3}}$

$-0.65 \mathrm{e}^{-} / \AA^{3}$ 